PHYSICAL REVIEW D 94, 055026 (2016)

\title{
Interplay of infrared divergences and gauge dependence of the effective potential
}

\author{
J. R. Espinosa, ${ }^{1,2}$ M. Garny, ${ }^{3}$ and T. Konstandin ${ }^{4}$ \\ ${ }^{1}$ Institut de Física d'Altes Energies (IFAE), The Barcelona Institute of Science and Technology (BIST), \\ Campus UAB, E-08193 Bellaterra (Barcelona), Spain \\ ${ }^{2}$ ICREA, Passeig Lluís Companys 23, 08010 Barcelona, Spain \\ ${ }^{3}$ CERN Theory Department, CH-1211 Geneva, Switzerland \\ ${ }^{4}$ DESY, Notkestraße 85, 22607 Hamburg, Germany \\ (Received 8 August 2016; published 20 September 2016)
}

\begin{abstract}
The perturbative effective potential suffers infrared (IR) divergences in gauges with massless Goldstones in their minima (like the Landau or Fermi gauges), but the problem can be fixed by a suitable resummation of the Goldstone propagators. When the potential minimum is generated radiatively, gauge independence of the potential at the minimum also requires resummation, and we demonstrate that the resummation that solves the IR problem also cures the gauge-dependence issue, showing this explicitly in the Abelian Higgs model in the Fermi gauge. In the process, we find an IR divergence (in the first derivative of the potential) specific to the Fermi gauge and not appreciated in the recent literature. We show that physical observables can still be computed in this gauge, and we further show how to get rid of this divergence by a field redefinition. All these results generalize to the Standard Model case.
\end{abstract}

DOI: 10.1103/PhysRevD.94.055026

\section{INTRODUCTION}

With the discovery of the Standard Model Higgs during the first LHC run [1], it quickly became clear that the precisely measured Higgs [2] and top [3] masses point to the possibility of a (very long-lived) metastable electroweak (EW) vacuum [4-9]. This fact has triggered a renewed interest in studies (and implications) of the possible metastability of the Standard Model EW vacuum (see, e.g., [10-12]).

The main tool for the study of this metastability is the perturbative effective potential [13], widely used for studies of spontaneous symmetry breaking. While the effective potential is an enormously useful tool in such studies, it (or the effective action from which it is derived) is not a physical observable and is subject to gauge dependence [13]. This is a well-known issue that has been studied extensively in the literature (see [14] for an incomplete list) and is of no serious concern: as long as one is calculating a physical observable (for example, the lifetime of the EW vacuum or other tunneling transitions; see e.g. [12,15-20]), the final answer should be gauge independent. ${ }^{1}$ However, this is not always straightforward to achieve in a concrete calculation: usually one must resort to truncations of the

\footnotetext{
${ }^{1}$ In fact, this requirement can be useful to check one is not missing some relevant effect.

Published by the American Physical Society under the terms of the Creative Commons Attribution 3.0 License. Further distribution of this work must maintain attribution to the author(s) and the published article's title, journal citation, and DOI.
}

perturbative expansion and this can jeopardize the gauge independence of the final result.

A well-known example of this kind of problem occurs in the Coleman-Weinberg model of radiative symmetry breaking [21] (as we review in subsection II C) or in the Standard Model (SM), as the instability that appears in the potential at high field values is generated radiatively. In order to have the gauge dependence of the potential under control in such cases, one must resort to resummations of series of corrections to the potential of arbitrarily high order, as nicely demonstrated in [19]. This type of resummation is reminiscent of the resummation of the Goldstone propagator needed to solve the infrared (IR) problem of the effective potential due to Goldstone contributions $[22,23]$ in those gauges that feature massless Goldstone bosons at the potential minima. One of the main results of this paper is that the resummation required to fix the IR problem automatically takes care of the resummation needed to control the gauge-dependence issues (subsection II C).

To check explicitly the gauge independence of the observables derived from the effective action one must resort to families of gauges. The most common gauge choices are the $R_{\xi}$ and Fermi gauges, both of which contain a gauge-fixing parameter (or parameters, that we will generically call $\xi$ ) that can be used to keep track of the gauge dependence. In this paper we follow a large fraction of recent literature and use the Fermi gauge for this purpose. In order to keep the analysis transparent, we work in the Abelian Higgs model (Sec. II A). The results we obtain can be extended to the SM in a straightforward way (Sec. V). 
In our analysis, we find that the Fermi gauge is afflicted by an IR divergence ${ }^{2}$ which is absent in the $R_{\xi}$ or Landau gauges (and can be traced back to the mixing of the Goldstone bosons with the gauge bosons). More specifically, the first derivative of the effective potential is logarithmically divergent for vanishing Goldstone mass (as happens in the broken minimum). Naively, this is a severe problem, since the minimum of the potential determines the vacuum of the system and is found by solving $\partial V / \partial h=0$. Furthermore, we show that this divergence persists even if the Goldstone propagator is resummed (Sec. II B). However, we also show that observables like the Higgs mass are IR finite (Sec. III A), and likewise the Nielsen identity still holds (Sec. III B). We take this good behavior as an indication that the Fermi gauge is not sick and we then present a way to obtain an explicitly IR-finite effective potential by a suitable rescaling of the Higgs field (Sec. IV). We draw some general details to a few Appendixes.

\section{IR PROBLEMS AND GAUGE DEPENDENCE}

Before discussing the gauge dependence of the effective potential we address the infrared problems associated with the presence of massless Goldstone modes at the potential minima. This problem was recognized in $[27,28]$, which identified IR divergent contributions to the effective potential from loops involving Goldstone bosons. The solution to this problem, in the Landau gauge, is simply to resum the Goldstone contributions by the appropriate shift of the Goldstone two-point function as was first proposed in [22,23] (see [29-31] for later developments and applications). This simple resummation makes the Landau-gauge potential and its first derivative IR finite. As an added bonus, it turns out (see subsection II C) that this resummation not only fixes the IR problems of the effective potential but it also resolves the issues with residual gauge dependence in those potentials that feature extremal points generated radiatively.

In what follows we want to apply this resummation prescription to the effective potential in the Fermi gauge, starting with the Abelian Higgs model and generalizing later on to the SM case. As we show explicitly below, the resummed potential is IR finite as desired. However, the first derivative, unlike what happened in the Landau gauge, is still IR divergent. We also provide a solution to this problem in Sec. IV. conclusions in Sec. VI and leave some more technical

\section{A. Abelian Higgs model in the Fermi gauge}

For simplicity, let us start the discussion of infrared problems in the Abelian Higgs model. The Lagrangian, in the Fermi gauge, reads

$\mathcal{L}=-\frac{1}{4} F_{\mu \nu} F^{\mu \nu}-\frac{1}{2 \xi}\left(\partial^{\mu} B_{\mu}\right)^{2}+\left|D_{\mu} \phi\right|^{2}+m^{2} \phi^{\dagger} \phi-\lambda\left(\phi^{\dagger} \phi\right)^{2}$,

where the covariant derivative for the charged "Higgs" field

$$
\phi=(h+i \chi) / \sqrt{2},
$$

is $D_{\mu}=\partial_{\mu}-i g B_{\mu}$. Without loss of generality we take the charge of $\phi$ under the $U(1)$ gauge symmetry to be unity and $F_{\mu \nu}$ is the corresponding field strength. The Lagrangian in Eq. (1) includes the gauge-fixing term, which corresponds to the so-called Fermi (or Lorentz) gauge, and depends on a free parameter, $\xi$. The limit $\xi \rightarrow 0$ corresponds to the Landau gauge.

The one-loop effective potential for this Abelian Higgs model was first derived long ago by Dolan and Jackiw, in their classic paper [32]. Its explicit expression requires knowing the masses in a generic field background $h$, in which one has ${ }^{3}$

$$
\begin{aligned}
G & \equiv m_{\chi}^{2}=-m^{2}+\lambda h^{2}, \\
H & \equiv m_{h}^{2}=-m^{2}+3 \lambda h^{2}, \\
B & \equiv m_{B}^{2}=g^{2} h^{2} .
\end{aligned}
$$

Using dimensional regularization, with $d=4-2 \epsilon$, the one-loop potential is then

$$
V_{1}=i \int \frac{d^{d} k}{(2 \pi)^{d}}\left(\sum_{\substack{\text { fermions } \\ \text { ghossts }}} \log \operatorname{det} i \mathcal{G}^{-1}-\frac{1}{2} \sum_{\text {bosons }} \log \operatorname{det} i \mathcal{G}^{-1}\right),
$$

where $i \mathcal{G}^{-1}$ denote the inverse of the propagators. For the Abelian Higgs model in the Fermi gauge in $d$ dimensions, one has contributions from transverse gauge bosons, Higgs and mixed Goldstone-longitudinal gauge bosons, giving

$\log \operatorname{det} i \mathcal{G}_{T}^{-1}=(d-1) \log \left(-k^{2}+B\right)$,

$\log \operatorname{det} i \mathcal{G}_{h}^{-1}=\log \left(-k^{2}+H\right)$,

$$
\begin{aligned}
\log \operatorname{det} i \mathcal{G}_{L}^{-1} & =\log \left[-k^{2}\left(k^{4}-k^{2} G+\xi B G\right)\right] \\
& =\log \left(-k^{2}+G_{+}\right)+\log \left(-k^{2}+G_{-}\right)+\log \left(-k^{2}\right),
\end{aligned}
$$

\footnotetext{
${ }^{2}$ Apparently this has escaped the attention of recent literature but was already known; see e.g. [24]. In the context of the Nielsen identity, the IR troubles with the Fermi gauge were remarked even earlier $[25,26]$.
}

\footnotetext{
${ }^{3}$ To simplify later expressions, here and in the following sections we use capital letters, as defined above, to denote field-dependent squared masses.
} 
with

$$
G_{ \pm}=\frac{1}{2}\left(G \pm \sqrt{G^{2}-4 \xi B G}\right)
$$

while the ghost-contribution is independent of the field value, $h$. Performing the momentum integrals, the final form of the renormalized effective potential, in $\overline{\mathrm{MS}}$ scheme, is

$$
\begin{aligned}
V_{1}= & \frac{\kappa}{4}\left[3 B^{2}\left(L_{B}-\frac{5}{6}\right)+H^{2}\left(L_{H}-\frac{3}{2}\right)\right. \\
& \left.+G_{+}^{2}\left(L_{G_{+}}-\frac{3}{2}\right)+G_{-}^{2}\left(L_{G_{-}}-\frac{3}{2}\right)\right],
\end{aligned}
$$

where $\kappa \equiv 1 /\left(16 \pi^{2}\right), L_{X} \equiv \log \left(X / \mu^{2}\right)$ and $\mu$ is the $\overline{\mathrm{MS}}$ renormalization scale. This agrees with the expression given in [33], translated to the Abelian case. The Landau-gauge limit corresponds to $\xi \rightarrow 0$, which gives $G_{+} \rightarrow G$ and $G_{-} \rightarrow 0$.

In analytical expansions below we will consider only the case $\xi>0$, which is usually better behaved than $\xi<0$ [33]. Eq. (6) shows that, for $\xi>0, G_{ \pm}$become imaginary in some field range even when $G>0$. However, the corresponding imaginary parts cancel each other out and the potential itself stays real. ${ }^{4}$

When one approaches the potential vacuum, $h \rightarrow v$ (with $v^{2}=m^{2} / \lambda$ at tree-level), the Goldstone mass goes to zero, $G \rightarrow 0$, and generically this induces IR divergences in the effective potential. In the Landau gauge, the potential $V$ first develops IR divergences at three-loop order [27]. The trouble comes from potential terms that are schematically of the form $\delta V \sim X^{2} \log G$, where $X$ is some mass-squared that is nonzero at the minimum of the potential. On the other hand, the first derivative $V^{\prime}$ of the potential is IR divergent already at two-loop order, from terms in the potential of the form $\delta V \sim X G \log G$. Finally, the second derivative $V^{\prime \prime}$ is IR divergent already at one loop, from a term $\delta V \sim G^{2} \log G$.

This infrared behavior is worse in the Fermi gauge than in the Landau gauge. The troublesome terms have the same origin but replacing $G$ by $G_{ \pm}$and the terms $\delta V \sim G_{ \pm}^{2} \log G_{ \pm}$cause $V^{\prime}$ to diverge already at one loop, due to the fact that $G_{+}^{\prime}$ diverges and $G_{+}^{\prime} G_{ \pm}$goes to a nonzero constant for $G \rightarrow 0$. To see this most clearly, notice that for $h \rightarrow v$ one has $G_{ \pm} \rightarrow \pm i \sqrt{\xi G B}$, so that $G_{ \pm}^{2} \log G_{ \pm} \sim X G \log G$. More precisely, the source of the trouble is the term

\footnotetext{
${ }^{4}$ This is most clearly seen in the unintegrated expression involving $\mathcal{G}_{L}^{-1}$ in Eq. (5). After the Wick rotation to Euclidean momentum $k^{2} \rightarrow-k_{E}^{2}$, one has $\log \left[-k^{2}\left(k^{4}-k^{2} G+\xi B G\right)\right] \rightarrow$ $\log \left[k_{E}^{2}\left(k_{E}^{4}+k_{E}^{2} G+\xi B G\right)\right]$ and the argument of the logarithm is positive for $\xi>0$ (and $G>0$ ).
}

$$
\delta V_{1}=-\frac{\kappa}{4} \xi G B \log \left(\frac{\xi G B}{\mu^{4}}\right) .
$$

In the following subsection, we apply to this Fermi gauge case the resummation proposed in [22,23] to cure such IR problems.

Let us close this subsection with a brief discussion of the gauge dependence of the potential in Eq. (7). In spite of the explicit $\xi$-dependence of the potential through its dependence on $G_{ \pm}$, it is well known that the value of the potential at its extremal points is guaranteed to be gauge invariant by the Nielsen identity [25,26,34]. At one-loop order, this is obviously the case of the potential in Eq. (7): the only dependence on $\xi$ of the potential appears in the $G_{ \pm}$terms and at the minimum $G \rightarrow 0$ one has $G_{ \pm} \rightarrow 0$, so that the $\xi$ dependence disappears.

\section{B. IR-Resummation}

The IR divergences in the effective potential are due to massless Goldstones, $G \rightarrow 0$, and come from diagrams with Goldstone bosons that carry small momentum, $k^{2} \sim G$. The worst divergences originate from those diagrams that have the largest possible number of Goldstone propagators with the same small momentum, and this number grows with higher-loop order. As shown in detail in Refs. [22,23] for the SM in the Landau gauge, these Goldstone divergences are spurious and can be resummed in a simple way by reorganizing the perturbative expansion. ${ }^{5}$ This is done by including the effect of self-energy diagrams on the Goldstone propagators, with $G \rightarrow \bar{G} \equiv G+\Pi_{g}$, where $\Pi_{g}$ is a well-defined radiative contribution to the Goldstone squared-mass that can be calculated perturbatively to the order needed. As explained in [23], $\Pi_{g}$ includes only contributions from heavy fields (that is, fields whose mass does not vanish when $G \rightarrow 0$ ) and hard Goldstones (with momentum $k^{2} \gg G$ ).

The effect of resummation in the Goldstone contribution to the one-loop potential in the Landau gauge is therefore the replacement ${ }^{6}$

$\delta_{G} V=\frac{\kappa}{4} G^{2}\left(L_{G}-3 / 2\right) \rightarrow \delta_{G} \bar{V}=\frac{\kappa}{4} \bar{G}^{2}\left(L_{\bar{G}}-3 / 2\right)$.

Expanding the latter expression perturbatively (in powers of $\kappa$ ) indeed reproduces the IR-divergent terms of the unresummed potential. In the unresummed perturbative expansion, the IR divergences occur at the field value for which $G \rightarrow 0$ : the location of the tree-level minimum. In the resummed potential, instead, possible IR divergences

\footnotetext{
${ }^{5} \mathrm{~A}$ similar problem (and solution) appears in $3 \mathrm{~d}$ (finite temperature) field theory studies of the EW phase transition [35].

${ }^{6}$ For our purposes in this paper, the one-loop resummed result (9) will be enough, but the resummation procedure can be extended to higher orders, see [23] for details.
} 
would occur at $\bar{G} \rightarrow 0$, which corresponds to the minimum of the radiatively corrected potential. However, for the resummed potential, $\bar{V}$ and $\bar{V}^{\prime}$ are IR finite and only the second derivative $\bar{V}^{\prime \prime}$ diverges for $\bar{G} \rightarrow 0$. However, this divergence is harmless and in fact required to get right the physical Higgs mass, as we discuss below.

The generic resummation of IR divergences just reviewed can also be applied to the Fermi gauge. The small complications associated with gauge bosonGoldstone mixed propagators can be circumvented in a simple way: add and subtract to the Lagrangian a term ${ }^{7}$ $-\Pi_{g} \chi^{2} / 2$, where $\Pi_{g}$ is the (zero-momentum) two-point function for the Goldstone field $\chi$ obtained as discussed above. The explicit expression for $\Pi_{g}$ in the Abelian model at one loop is

$$
\Pi_{g}=3 \kappa\left[g^{2} B\left(L_{B}-\frac{1}{3}\right)+\lambda H\left(L_{H}-1\right)\right],
$$

which can be directly obtained from the contribution of $B$ and $H$ to the one-loop potential in Eq. (7), remembering that the Goldstone mass is given by $(\partial V / \partial h) / h$. The added term is treated as shifting the Goldstone mass that appears in propagators, with $G \rightarrow \bar{G} \equiv G+\Pi_{g}$, while the subtracted term is treated as a counterterm. After this shift, the two field-dependent masses corresponding to the mixed Goldstone-gauge boson sector are given by

$$
\bar{G}_{ \pm} \equiv \frac{1}{2}\left(\bar{G} \pm \sqrt{\bar{G}^{2}-4 \xi \bar{G} B}\right),
$$

to be compared with Eq. (6). The expression for the oneloop effective potential of Eq. (7) with this resummation implemented is obtained from the unresummed one simply by the replacement $G_{ \pm} \rightarrow \bar{G}_{ \pm}$.

Does this resummation achieve the desired cure of the IR divergence problems of the effective potential also in the Fermi gauge? While it is clear that the resummed potential is finite in the $\bar{G} \rightarrow 0$ limit, its first derivative (which is the crucial quantity to determine the location of the potential minimum) is not finite even after resummation. The unresummed term that causes the IR divergence in $V^{\prime}$ is of the form $\delta V \sim X G \log G$ as discussed at the end of the previous section and resummation simply changes this to $\delta \bar{V} \sim X \bar{G} \log \bar{G}$, which still gives a divergent $\bar{V}^{\prime}$. Note that the divergence in $\bar{V}^{\prime}$ is $\xi$ dependent and goes away for $\xi=0$. This divergence, which can be formally translated into a divergence in the one-loop vacuum expectation value (VEV), had been pointed out before (see, e.g., [24]) but seems to have gone unnoticed in more recent literature. Before discussing the solution to the previous problem (deferred to subsection IV below), it is instructive to

\footnotetext{
${ }^{7} \mathrm{~A}$ more sophisticated procedure is described in subsection IIIBl; see, in particular, footnote 13 .
}

compare the resummation performed above with the resummation discussed in Ref. [19] to solve a different issue.

\section{IR resummation eliminates residual gauge dependence}

Suppose we are interested in the gauge dependence of the effective potential close to the electroweak vacuum. The perturbative counting is the conventional loop counting, with $g^{2} \sim \lambda$. The naive expectation is that, using this counting, a consistent expansion of the effective action will fulfill the Nielsen identity [25] and hence provide gauge-independent observables. As we have described above, potentially this requires resummation of certain classes of diagrams $[18,22,23]$, most notably two-particlereducible diagrams of light particles in those gauges (like the Landau or Fermi gauge) in which the Goldstone boson is massless at the minimum.

Things are different if the electroweak vacuum is generated radiatively. The best known example is the Coleman-Weinberg model [21], which is nothing but the Abelian Higgs model with a massless scalar $h$ [that is, $m^{2}=0$ in the Lagrangian of Eq. (1)]. Famously, the interest of the model lies in the possibility of radiatively breaking the $U(1)$ gauge symmetry (a paradigmatic example of dimensional transmutation). For studies of the gauge dependence of the effective potential, the difficulty with this model was recognized already in Refs. [25,34]: the minimum appears through the balance between the treelevel quartic coupling $\lambda$ and the one-loop radiative corrections, of order $\hbar g^{4}$, so that for power counting one should use $\lambda \sim \hbar g^{4}$. This jeopardizes the usual fixed-order loop expansion of the effective potential: one-loop terms of order $\hbar \lambda g^{2}$ are of the same order as two-loop terms of order $\hbar^{2} g^{6}$ or three-loop terms of order $\hbar^{3} g^{10} / \lambda$, and this should be taken into account when showing the gauge independence of the value of the potential at its minimum, that would have a residual gauge dependence if calculated at a fixed order in perturbation theory. It was also clear [36] that a resummation that reorganizes the perturbative expansion would get rid of this problem and this has been shown explicitly to two-loop order in Ref. [19].

A similar situation arises in the SM effective potential at very high values of the Higgs field, when an instability is generated by radiative corrections. Previous work in the Fermi gauge has studied the gauge dependence of the potential at such high field values [19,33], at which one can neglect the explicit mass term in the Lagrangian (that is of electroweak scale size) and use the counting $\lambda \simeq \hbar g^{4} \simeq \hbar y_{t}^{4}$. This simplifies the analysis of the effective action, since the one-loop corrections to the effective potential from the Goldstone bosons scale as $\lambda^{2}$ and hence are of the same order as three-loop contributions from the gauge sector $\sim g^{8}$. Thus, in a two-loop analysis up to order 
$g^{6} \sim \lambda^{3 / 2}$ some of the IR issues do not enter yet. But even in this simplified case, the same subtleties discussed for the Coleman-Weinberg model concerning gauge dependence remain, as emphasized in [19].

We now show that the resummation required to cure the IR problems of the potential discussed in the previous subsection automatically takes care of this gauge issue. To ease the comparison, note that the notation of [19] for the Coleman-Weinberg model in the Fermi gauge, differs from ours: $h$ is called $\phi, g$ is $e$, and our $\lambda$ is replaced by $\lambda / 6$. The resummation shift in the Goldstone mass, $G=\lambda h^{2} \rightarrow \bar{G}=G+\Pi_{g}$, corresponds to the shift $\lambda \rightarrow \bar{\lambda}(h) \equiv \lambda-\hat{\lambda}(h)$, where

$$
\hat{\lambda}(h) \equiv 36 \kappa g^{4}\left(1-3 L_{B}\right),
$$

with $\kappa=\hbar /\left(16 \pi^{2}\right)$ and $L_{B}=\log \left(g^{2} h^{2} / \mu^{2}\right)$. We have used here the results of the previous section setting $m^{2}=0$ and neglecting $\lambda^{2}$ corrections in Eq. (10), which are of higher order as $\lambda \sim \hbar g^{4}$.

The expression for the resummed two-loop potential is quite simple:

$$
\begin{aligned}
\bar{V}_{2}(h)= & \frac{1}{4} \lambda h^{4}+\frac{3 \kappa}{4} g^{4} h^{4}\left(L_{B}-\frac{5}{6}\right) \\
& -\frac{\kappa}{4} \xi g^{2} \bar{\lambda}(h) h^{4}\left(\log \frac{\xi g^{2} \bar{\lambda}(h) h^{4}}{\mu^{4}}-3\right) \\
& +\kappa^{2} g^{6} h^{4}\left(\frac{5}{2} L_{B}^{2}-\frac{31}{3} L_{B}+\frac{71}{6}\right) .
\end{aligned}
$$

Some comments are in order: (1) This expression packages in a compact way the result for the two-loop potential given in [19], Eqs. (6.16-17). (2) The resummation performed to deal with IR divergences generates directly all the two-loop terms necessary to check gauge independence, without residual gauge dependence left. (3) The gauge independence of the potential value at the minimum is straightforward to see as the result of the minimum corresponding (at one-loop order) to $\bar{\lambda}(v)=0$.

The resummed expression given in Eq. (13) also sheds some light on the IR problem in $V^{\prime}$ discussed in the previous subsection as specific to the Fermi gauge. Indeed there is a ( $\xi$-dependent) logarithmic divergence in $d V / d h \sim \kappa \xi g^{2}(d \bar{\lambda} / d h) \log \bar{\lambda}$ for $\bar{\lambda} \rightarrow 0$. Moreover, notice that $d \bar{\lambda} / d h=-d \hat{\lambda} / d h \sim \kappa g^{4}$, so that the IR divergence is a two-loop effect of order $\kappa g^{6}$, and no other terms of that order in the potential (13) could cancel out such divergence.

Nevertheless, this obstruction looks strange, given the fact that the first derivative of the potential $V$, to whatever precision it is calculated, and the Goldstone mass calculated to the same precision, are related ${ }^{8}$ as

\footnotetext{
${ }^{8}$ As the effective potential in a generic background is a function of $|\phi|^{2}=\left(h^{2}+\chi^{2}\right) / 2$.
}

$G=(d V / d h) / h$. In a consistent calculation there seems to be no room for a zero in $G$ causing a divergence in $d V / d h$. In view of this, we could consider Eq. (13) as the correct expression for the two-loop resummed potential but with $\bar{\lambda}$ to be specified in a self-consistent way. Then we could use the relation $\bar{G}=\bar{\lambda}(h) h^{2}=\left(\partial \bar{V}_{2} / \partial h\right) / h$ to define $\bar{\lambda}$. At two loops, with the approximations used, one gets

$$
\begin{aligned}
\bar{\lambda}(h)= & \lambda+\kappa g^{4}\left(3 L_{B}-1\right)+2 \kappa \xi \lambda g^{2}\left(1-L_{B}\right) \\
& +\kappa^{2} g^{6}\left\{\frac{2}{3}\left[40-\left(47-15 L_{B}\right) L_{B}\right]+\xi\left[1+\left(5-6 L_{B}\right) L_{B}\right]\right\} \\
& -\kappa \xi g^{2}\left[\lambda+\frac{1}{2} \kappa g^{4}\left(1+6 L_{B}\right)\right] \log \left(\xi \bar{\lambda} / g^{2}\right) .
\end{aligned}
$$

However, this definition of $\bar{\lambda}(h)$ is problematic for $\bar{\lambda} \rightarrow 0$ as the prefactor of the last logarithm does not go to zero in that limit. Another way of stating the problem is this: fix the values of $\lambda, g$ and $\xi$ at some given $\mu$. Using Eq. (14) as the definition of the function $\bar{\lambda}(h)$, we see that, when $\bar{\lambda}$ gets close to zero, $\log \bar{\lambda}$ blows up and destroys perturbativity.

The ultimate root of this IR divergence is the pole of order $p^{4}$ in the mixed propagator of Goldstone bosons and longitudinal gauge bosons, which shows up clearly in their contribution to the effective potential; see Eq. (5). ${ }^{9}$ Moreover, this problem persists even if no perturbative expansion is used (as we show in Appendix A using the Ward identity). This mixed propagator is a specific feature of the Fermi gauge which explains why such IR divergence is absent in the Landau gauge $(\xi \rightarrow 0)$ or in the background $R_{\xi}$ gauges.

Naively, one might be tempted to conclude that there is no acceptable description of symmetry breaking within perturbation theory in the Fermi gauge (unless $\xi=0$, which is the Landau gauge). Nevertheless, as physical quantities cannot depend on the gauge parameter $\xi$, one could expect that the $\xi$-dependent IR divergence will cancel out when calculating observables. In the following section, we show that this expectation is fulfilled for the physical Higgs mass.

\section{PHYSICAL RESULTS IN THE FERMI GAUGE}

In this section, we show how physical information can be extracted in the Fermi gauge even though the effective potential has no well-defined minimum at one-loop order. First we discuss the mass of the Higgs boson and then how to make sense of the Nielsen identity in spite of the IR divergences that afflict the Fermi gauge.

\footnotetext{
${ }^{9} \mathrm{~A}$ similar $p^{4}$ pole appears in supersymmetric QED (in the propagator of the lowest component of the vector superfield), leading to IR divergences. For a recent discussion, see [37].
} 


\section{A. The physical Higgs mass}

We show now how the physical Higgs mass is free of IR divergences even when one calculates it in the Fermi gauge. We go back here to the general Abelian Higgs model, with nonzero $m^{2}$. The physical Higgs mass is defined as the pole of the Higgs propagator. Calculated at one-loop order, it is

$$
\begin{aligned}
M_{h}^{2} & =V_{0}^{\prime \prime}(v)+\left.\Sigma\left(M_{h}^{2}\right)\right|_{0} \\
& =-m^{2}+3 \lambda v_{0}^{2}+6 \lambda v_{0} \delta v+\left.\Sigma\left(M_{h}^{2}\right)\right|_{0} .
\end{aligned}
$$

Here, field derivatives are represented by primes; the one-loop VEV is $v=v_{0}+\delta v$ with $v_{0}=\sqrt{m^{2} / \lambda}$ the tree-level vacuum expectation value [calculated with the tree-level potential $\left.V_{0}(h)\right]$ and $\delta v$ the one-loop correction to it, given by

$$
\delta v=-\left.\frac{1}{2 \lambda v_{0}^{2}} \frac{\partial V_{1}}{\partial h}\right|_{0}
$$

Finally, $\Sigma\left(p^{2}\right)$ is the one-loop 1PI two-point function of the Higgs, with external momentum $p$. With $\left.\right|_{0}$ we indicate that the limit $h \rightarrow v_{0}$ is taken, which is appropriate at one-loop order. All the parameters entering Eq. (15) are already the renormalized ones.

The explicit result in the Fermi gauge, using dimensional regularization, $L_{X} \equiv \log \left(X / \mu^{2}\right)$, and taking the limit $h \rightarrow v_{0}$ everywhere except in the logarithmically divergent terms, yields (see Appendix B for details)

$$
\begin{aligned}
\left.6 \lambda v_{0} \delta v\right|_{0}= & 3 \kappa v_{0}^{2}\left\{6 \lambda^{2}\left(1-L_{H}\right)+g^{4}\left(1-3 L_{B}\right)\right. \\
& \left.-\frac{1}{2} \lambda g^{2} \xi\left[2-\log \left(\frac{\xi G B}{\mu^{4}}\right)\right]\right\}\left.\right|_{0},
\end{aligned}
$$

and

$$
\begin{aligned}
\left.\Sigma\left(M_{h}^{2}\right)\right|_{0}= & \kappa v_{0}^{2}\left\{3 g^{4}\left(1+L_{B}\right)+\lambda g^{2}\right. \\
& \times\left[2+3 \xi-2 L_{B}-\frac{3}{2} \xi \log \left(\frac{\xi G B}{\mu^{4}}\right)\right] \\
& +6 \lambda^{2}\left(\pi \sqrt{3}-7+4 L_{H}\right) \\
& \left.-2\left(\lambda^{2}-2 \lambda g^{2}+3 g^{4}\right) B_{0}^{R}(B, B, H)\right\}\left.\right|_{0},
\end{aligned}
$$

where we have left unevaluated the (renormalized) oneloop integral,

$$
\begin{aligned}
& B_{0}^{R}\left(m_{1}^{2}, m_{2}^{2}, p^{2}\right) \\
& \quad=-\int_{0}^{1} d x \log \left[\frac{m_{1}^{2}(1-x)+m_{2}^{2} x-x(1-x) p^{2}-i \varepsilon}{\mu^{2}}\right],
\end{aligned}
$$

and we leave explicit the terms that cause a divergence in the $G \rightarrow 0$ limit. In the sum that gives $M_{h}^{2}$, the terms involving $\xi$ in (17) and (18) cancel, as expected for a physical quantity [38]. Furthermore, one can check that the result above for $M_{h}^{2}$ agrees with the SM result calculated in the Landau gauge ${ }^{10}[4,39]$ and Feynman gauge [40], appropriately reduced to the Abelian Higgs model.

Note that the terms that diverge logarithmically for $h \rightarrow v_{0}$ (i.e., for $G \rightarrow 0$ ), that is, the IR divergence from the shift in the Higgs VEV (17) and from the self-energy (18), cancel in the sum, and one has

$$
-3 \frac{V_{1}^{\prime}}{v_{0}}+\Sigma\left(M_{h}^{2}\right) \Rightarrow \text { IR finite. }
$$

Therefore, even though the Higgs VEV (not an observable) diverges, observable physical quantities as the Higgs mass are finite. ${ }^{11}$ We also note that the imaginary part of the pole of the Higgs propagator (15), that is related to the Higgs width, is gauge independent. Specifically, after the cancellation of the terms involving $\xi$ in (17) and (18), the only contribution to the imaginary part arises from the last term in (18) that involves the one-loop integral (19). This term is independent of $\xi$, and has a nonzero imaginary part for $H>4 B$ (that is, $m_{H}>2 m_{B}$ ), corresponding to the decay of the Higgs into a pair of gauge bosons.

We close this subsection with am illustrative comparison of the IR divergences in the 1PI two-point function $\Sigma\left(p^{2}\right)$ between the Landau and Fermi gauges. In the Landau gauge, the IR divergent terms at one-loop are

$$
\begin{aligned}
\kappa^{-1} \Sigma_{\mathrm{IR}}\left(p^{2}\right) & =0, \quad\left(\text { for } p^{2} \neq 0\right) \\
\kappa^{-1} \Sigma_{\mathrm{IR}}(0) & =2 \lambda^{2} v^{2} L_{G} .
\end{aligned}
$$

This divergent structure is correlated with the fact that, in this gauge, $V^{\prime}$ is IR finite and $V^{\prime \prime}$ IR divergent, as $V_{1}^{\prime \prime}=\Sigma(0)$.

In the Fermi gauge, instead, we have

$$
\begin{aligned}
\kappa^{-1} \Sigma_{\mathrm{IR}}\left(p^{2}\right)= & \frac{\xi B L_{G}}{2 v^{2}}\left[\frac{1}{p^{2}}\left(H-p^{2}\right)^{2}\left(1+\frac{\xi B}{p^{2}}\right)-5 H+2 p^{2}\right], \\
& \left(\text { for } p^{2} \neq 0\right) \\
\kappa^{-1} \Sigma_{\mathrm{IR}}(0)= & -\lambda^{2} v^{2} \xi \frac{B}{G}-\frac{3 \pi}{4} \lambda^{2} v^{2} \sqrt{\frac{\xi B}{G}}+\left(\lambda^{2} v^{2}-\frac{5}{2} \lambda \xi B\right) L_{G} .
\end{aligned}
$$

\footnotetext{
${ }^{10}$ Starting from Eqs. (2.12)-(2.13) of [4], one gets $M_{h}^{2}=2 \lambda v_{0}^{2}+4 \lambda v_{0} \delta v^{(\xi=0)}+\delta_{1} M_{h}^{2}$, where $\delta v^{(\xi=0)}$ is the shift of the minimum in the Landau gauge. Taking into account the different conventions used, and translating to the Abelian case $\left(y_{t} \rightarrow 0, g \rightarrow 0, g^{\prime 2} \rightarrow g^{2} / 4, M_{Z}^{2} \rightarrow B\right.$, and ignoring terms involving $M_{W}$ ) there is agreement.

${ }^{11}$ This cancellation is reminiscent of a similar cancellation that takes place in the computation of the Higgs mass as $M_{h}^{2}=V^{\prime \prime}+\Sigma\left(M_{h}^{2}\right)-\Sigma(0)$ where $V$ is now the full potential and the $\Sigma$ terms take into account that the mass is defined on-shell and not at $p^{2}=0$. At one loop, $V_{1}{ }^{\prime \prime}$ has a logarithmic IR divergence that is precisely canceled by $\Sigma_{1}\left(M_{h}^{2}\right)-\Sigma_{1}(0)$, as is obvious from the fact that $V_{1}^{\prime \prime}$ is nothing but $\Sigma_{1}(0)$.
} 
We see that $\Sigma$ is IR divergent even on-shell, and this is correlated (in order to get an IR finite Higgs mass as discussed above) with the IR divergence in $V^{\prime}$ present in this gauge, see Eq. (20). We also see that the $\xi \rightarrow 0$ limit of $\Sigma_{\text {IR }}(0)$ differs from the Landau gauge result showing explicitly that the limits $\xi \rightarrow 0$ and $p^{2} \rightarrow 0$ do not commute.

\section{B. Nielsen identity}

The Nielsen identity $[25,26,34]$ plays a central role for the gauge (in)dependence of the potential. In this subsection, we examine how the IR divergence in $V^{\prime}$ affects this identity, which reads

$$
\xi \frac{\partial V}{\partial \xi}+C \frac{\partial V}{\partial h}=0
$$

where the function $C$ is the constant background limit of a function $C(x)$ (which enters the Nielsen identity for the effective action) that in the Fermi gauge reads

$$
C(x)=\frac{i g}{2} \int d^{4} y\left\langle c(x) \chi(x) \bar{c}(y) \partial_{\mu} B^{\mu}(y)\right\rangle,
$$

with $c, \bar{c}$ the ghost fields. The (renormalized) one-loop result reads

$$
C_{1}=\frac{\kappa \xi B}{2 v\left(G_{+}-G_{-}\right)}\left[G_{+}\left(L_{G_{+}}-1\right)-G_{-}\left(L_{G_{-}}-1\right)\right] .
$$

Taking the limit $h \rightarrow v$ (or $G \rightarrow 0$ ), we find

$$
C_{1} \rightarrow-\frac{1}{2} \kappa \xi g^{2} v\left[1-\frac{1}{2} \log \left(\frac{\xi G B}{\mu^{4}}\right)\right],
$$

which is logarithmically IR divergent for $G \rightarrow 0 .{ }^{12}$ However, evaluating the Nielsen identity perturbatively, at one loop one gets (with primes denoting field derivatives):

$$
\xi \frac{\partial V_{1}}{\partial \xi}+C_{1} V_{0}^{\prime}=0
$$

and the one-loop product $C_{1} V_{0}{ }^{\prime} \propto C_{1} G$ goes to zero for $G \rightarrow 0$. This means that the value of $V$ at the minimum, or more precisely $\left.V_{1}\right|_{v}$, is gauge independent, as it should be.

The fact that the (one-loop) Nielsen identity (valid for arbitrary field values) is IR finite implies that all the identities derived from it by taking field derivatives are also IR finite even if individual terms diverge. For example, taking one field derivative of the Nielsen identity gives

\footnotetext{
${ }^{12}$ This was noticed already in [25] and was later taken as reason to avoid the use of the Fermi gauge, e.g., in [26].
}

$$
\xi \frac{\partial V^{\prime}}{\partial \xi}+C V^{\prime \prime}+C^{\prime} V^{\prime}=0
$$

When evaluated close to the potential minimum, $h=v$, the first term essentially determines the gauge dependence of the location of that minimum. One can then check that the IR divergences in the first two terms cancel each other. Naively, one may think that the last term vanishes at $v$. However, this is not the case because $C^{\prime} \propto 1 / G$ such that the product $C^{\prime} V_{0}^{\prime}$ does not vanish in the minimum.

A similar discussion applies to the Nielsen identity for the kinetic term $Z(h)\left(\partial_{\mu} h\right)^{2} / 2$ in the effective action (derived in Appendix $C$ for the Fermi gauge at one loop). As in the Landau gauge, $Z(h)$ is IR divergent close to the broken phase minimum. The enhanced IR sensitivity of $Z$ can be attributed to the gradient expansion around homogeneous field configurations as well as the vanishing Goldstone boson mass in the broken minimum, that occurs both in the Landau and Fermi gauges. Nevertheless, the IR sensitivity of the coefficients appearing in the Nielsen identity for $Z$ matches precisely those that are present in $\xi \partial Z / \partial \xi$, and the Nielsen identity holds at one loop for all field values. In addition, as discussed above, all IR divergences cancel for physical observables.

We conclude that the Nielsen identity is fulfilled order by order in the perturbative expansion and that, for the effective potential, IR effects explicitly cancel. Finally, it can be shown that the identity will also hold after including resummation effects in a consistent way. For instance, the one-loop resummed potential fulfills the identity as the replacement $G \rightarrow \bar{G}=G+\Pi_{g}$ (or $G_{ \pm} \rightarrow \bar{G}_{ \pm}$), with a $\xi$-independent $\Pi_{g}$, does not interfere with the structure of the identity if one uses, to be consistent, that $V^{\prime}=h \bar{G}$. In fact, there is a systematic way to consistently maintain the Nielsen identity order by order even when including IR resummation in the following way: Add to the Lagrangian $-\Pi_{g} \phi^{\dagger} \phi+\Pi_{g} \phi^{\dagger} \phi=0$, and absorb the first term into a shift of the quadratic term $-m^{2} \rightarrow-m^{2}+\Pi_{g}$ that enters in the Goldstone and Higgs mass. On the other hand, the second term is treated perturbatively as a one-loop counterterm, i.e., $\mathcal{O}(\hbar)$. This procedure implements the replacement $G \rightarrow \bar{G}$ in the one-loop expressions and, in addition, also the Higgs mass gets shifted. This ensures that the potential at order $\hbar^{0}$ fulfills $V^{\prime}=h \bar{G}$, and therefore the Nielsen identity is precisely fulfilled. ${ }^{13}$ On the other hand, since $\phi^{\dagger} \phi=\left(h^{2}+\chi^{2}\right) / 2$ this also introduces a shift in the Higgs mass parameter $H \rightarrow H+\Pi_{g}$ that appears in the Higgs propagator in loop diagrams. However, this shift is

\footnotetext{
${ }^{13}$ Note that for this to be true it is not sufficient to add and subtract only a mass term for the Goldstone boson $-\left(\Pi_{g}-\Pi_{g}\right) \chi^{2} / 2$. The reason is that this operator is not gauge invariant, and therefore jeopardizes the Nielsen identity once the first term is resummed while the second is treated as a one-loop counterterm. Using instead the operator $\phi^{\dagger} \phi$ solves this problem.
} 
perturbatively small (since the Higgs mass does not vanish close to the broken phase minimum) and therefore it is canceled by the corresponding counterterm contributions up to terms of higher order in perturbation theory.

\section{SOLUTIONS TO THE IR PROBLEM IN THE FERMI GAUGE}

Although, as we have seen, IR divergences should not affect observables, it can be convenient to get rid of the IR divergences also in intermediate results. In particular, it is more satisfactory to have an effective potential whose first derivative is IR finite and does not suffer from a formally infinite shift in the location of its minimum. ${ }^{14}$ With such goal in mind, an obvious solution to try is to absorb the infinite shift in a field redefinition.

Notice that it would be ill advised to keep the Higgs VEV finite by suitable renormalization conditions on the masses and/or couplings. The observables are invariant under gauge changes for fixed bare couplings while the Higgs VEV is not. From this point of view, if the bare couplings are fixed in the Landau gauge, the IR issue in the Higgs VEV seems a necessity in the Fermi gauges with $\xi \neq 0$. In contrast, the wave function renormalization and field redefinitions in general are unobservable.

\section{A. Field redefinition}

Let us see then what field redefinition would be needed to make $V^{\prime}$ IR finite. Consider a field redefinition of the form

$$
h \rightarrow h+\hbar F(h)
$$

where we have included an explicit factor $\hbar$ to indicate that the shift is of one-loop order. Such field redefinitions modify the form of the Lagrangian without affecting the physics (more precisely, without modifying $S$-matrix elements [41]). The change induced by the shift in Eq. ([41]) on the potential is

$V=V_{0}+\hbar V_{1}+\mathcal{O}\left(\hbar^{2}\right) \rightarrow V=V_{0}+V_{0}{ }^{\prime} \hbar F+\hbar V_{1}+\mathcal{O}\left(\hbar^{2}\right)$,

with primes denoting field derivatives, as usual. There is some freedom in choosing the function $F(h)$ so that it cancels out the IR divergence in $V_{1}{ }^{\prime}$, because the cancellation should occur at a single point in field space. The IR divergence comes from the potential term $\delta V_{1}=$ $-(\kappa / 4) \xi G B \log \left(\xi G B / \mu^{4}\right)$ and we have the choice of removing this, or just the part that goes like $\log \left(G / \mu^{2}\right)$ or the full Goldstone contribution, etc. We do not commit at

\footnotetext{
${ }^{14}$ The shift of the VEV is not actually infinite. What happens is that the potential develops a cusp at $\vec{\lambda}=0$, which is therefore a bad starting point for the perturbative expansion.
}

this point with such choices and write generically the term to be removed as $\delta V_{1}=-(\kappa / 4) \xi G B \log (G / X)$ with $X$, a quantity of dimension mass squared, to be chosen later on. Simple inspection of the first derivative of the shifted potential in Eq. (30) shows that $F(h)$ evaluated at $h=v$ should satisfy

$$
F(v)=\left.\frac{\kappa}{4} \xi g^{2} v \log \frac{G}{X}\right|_{v} .
$$

Once we get an IR finite $V_{1}{ }^{\prime}$, it can no longer cancel the IR divergence of $\Sigma\left(M_{h}^{2}\right)$ as needed to get $M_{h}^{2}$ finite, see Eq. (20) and the discussion at the end of subsection III A. However, this causes no problem as we should also consider the impact of the field shift ([41]) on the kinetic term for $h$,

$$
\frac{1}{2}\left(\partial_{\mu} h\right)^{2} \rightarrow \frac{1}{2}\left(\partial_{\mu} h\right)^{2}+\hbar F^{\prime}\left(\partial_{\mu} h\right)^{2},
$$

that modifies the Higgs pole mass equation (15) as

$$
\left(1+2 F^{\prime}\right) M_{h}^{2}=\left[V_{0}+F V_{0}^{\prime}\right]^{\prime \prime}(v)+\left.\Sigma\left(M_{h}^{2}\right)\right|_{0} .
$$

In this equation we have treated the $F$-shift terms in (30) and (32) as modifying the tree-level Lagrangian (even though they are shifts of order $\hbar$ ). The one-loop radiative corrections calculated with this shifted Lagrangian are the same as before so that $\left.\Sigma\left(M_{h}^{2}\right)\right|_{0}$ above is the same as in (15), and the one-loop VEV $v$ is the minimum of the full oneloop potential (30). Explicitly,

$$
v=v_{0}+\delta_{F} v+\delta v,
$$

with

$$
v_{0}^{2}=\frac{m^{2}}{\lambda}, \quad \delta_{F} v=-F, \quad \delta v=-\frac{V_{1}^{\prime}}{V_{0}^{\prime \prime}},
$$

so that $\delta v$ is the same as in (15) but now $\delta_{F} v+\delta v$ is IR finite by construction. More explicitly, expanding $v$ in Eq. (33) and keeping terms up to $\mathcal{O}(\hbar)$ we get

$$
\begin{aligned}
\left(1+2 F^{\prime}\right) M_{h}^{2}= & V_{0}^{\prime \prime}\left(v_{0}\right)+\left(\delta_{F} v+\delta v\right) V_{0}^{\prime \prime \prime}+2 F^{\prime} V_{0}^{\prime \prime} \\
& +F V_{0}^{\prime \prime \prime}+\left.\Sigma\left(M_{h}^{2}\right)\right|_{0} .
\end{aligned}
$$

The $F^{\prime}$ terms cancel out and we end up with

$M_{h}^{2}=2 \lambda v_{0}^{2}+3 \lambda v_{0}\left(\delta_{F} v+\delta v\right)+\left[3 \lambda v_{0} F+\left.\Sigma\left(M_{h}^{2}\right)\right|_{0}\right]$.

We see explicitly that $M_{h}^{2}$ is exactly the same as the one in (15) as $3 \lambda v_{0}\left(\delta_{F} v+F\right)=0$. Moreover, the IR divergence of $\left.\Sigma\left(M_{h}^{2}\right)\right|_{0}$ is precisely canceled by the $3 \lambda v_{0} F$ term. Therefore, we see explicitly that the physical Higgs mass, 
an observable, is not affected by our field redefinition, as expected.

A simple and convenient choice of $F(h)$ that satisfies the condition (31) corresponds to the field redefinition,

$$
h \rightarrow h+\frac{\kappa}{4} \xi g^{2} h \log \frac{\xi G B}{\mu^{4}},
$$

corresponding to the choice $X=\mu^{4} /(\xi B)$. This field redefinition, being $\mu$-dependent, modifies the wavefunction renormalization of the field, encoded in the anomalous dimension $\gamma \equiv d \log h / d \log \mu$. One gets that the one-loop $\gamma$ is shifted as

$\gamma_{1}=\kappa g^{2}(3-\xi) \rightarrow \gamma_{1}=\kappa g^{2}(3-\xi)+\kappa \xi g^{2}=3 \kappa g^{2}$,

and the $\xi$ dependence drops (which can be useful to reduce the gauge dependence of the potential). However, as we have already mentioned, one is not forced to this choice of $X$, and if one takes instead a $\mu$-independent $X$ (say $X=B$ ), the one-loop anomalous dimension of $h$ will not change.

The potential expressed in terms of the shifted field [after $\left.h \rightarrow h+(\kappa / 4) \xi g^{2} h \log (G / X)\right]$ reads, at one loop:

$$
\begin{aligned}
V= & -\frac{1}{2} m^{2} h^{2}+\frac{\lambda}{4} h^{4} \\
& +\frac{\kappa}{4}\left[3 B^{2}\left(\log \frac{B}{\mu^{2}}-\frac{5}{6}\right)+H^{2}\left(\log \frac{H}{\mu^{2}}-\frac{3}{2}\right)\right] \\
& +\frac{\kappa}{8} G\left[G\left(\log \frac{\xi G B}{\mu^{4}}-3\right)-2 \xi B\left(\log \frac{\xi X B}{\mu^{4}}-3\right)\right. \\
& \left.+\left(G_{+}-G_{-}\right) \log \frac{G_{+}}{G_{-}}\right] .
\end{aligned}
$$

One can explicitly check that $V^{\prime}$ is now finite for $G \rightarrow 0$ and the one-loop shift of the VEV is IR finite. The field redefinition we have performed is reminiscent of the field redefinition proposed in [42] to obtain a $\xi$-independent potential (which in practice is equivalent to going to the Landau gauge) or in [12] to make the field canonical and reduce the $\xi$ dependence of the potential. Our aim here is different: we just want to remove the IR problem but leave the $\xi$ dependence as we want to study the gauge (in) dependence of different quantities. Still, one could argue that our field redefinitions either fix the gauge (if all $\xi$ dependence is gone) or amount to using a different gauge fixing (in some sense intermediate between the Fermi and Landau gauges).

Let us consider next the IR structure of the Nielsen identity after the field redefinition in (38). At one loop, the identity takes the form in Eq. (27). As the field shift sends $V_{1} \rightarrow V_{1}+F V_{0}^{\prime}$ we immediately deduce that $C_{1} \rightarrow C_{1}-\xi \partial F / \partial \xi$

$$
C_{1} \rightarrow C_{1}-\frac{\kappa}{4} \xi g^{2} h\left(\log \frac{G}{X}+1\right),
$$

so that the Nielsen identity is respected. From the IR limit in Eq. (26) we see that the new $C_{1}$ above is instead IR finite. Thus, the same field redefinition that removes the IR divergence from the first derivative of the effective potential also removes the IR divergence in the Nielsen coefficient. In addition, when taking a derivative of the shifted Nielsen identity with respect to the shifted field, the contribution $C^{\prime} V_{0}{ }^{\prime}$ actually vanishes for $h \rightarrow v_{0}$, in accordance with naive expectations.

\section{B. IR regulator}

Above we have shown how to use a field redefinition to get a potential with a well-behaved (i.e., IR finite) first derivative. Instead, we could simply regulate the IR divergences, checking at the end of the calculations that physical quantities are independent of the IR regulator. A simple way of doing this is to use the Fukuda-Kugo gauge [34],

$$
\mathcal{L}_{g f}=-\frac{1}{2 \xi}\left(\partial^{\mu} B_{\mu}+\mu_{\mathrm{IR}} \chi\right)^{2},
$$

that leads to the masses

$G_{ \pm}=\frac{1}{2}\left[G+2 \mu_{\mathrm{IR}} m_{B} \pm \sqrt{G^{2}-4\left(\xi B-\mu_{\mathrm{IR}} m_{B}\right) G}\right]$

which tend to $\mu_{\mathrm{IR}} m_{B}$ when $G \rightarrow 0$. Therefore, in this gauge $\mu_{\mathrm{IR}}$ acts as an IR regulator of the divergences that afflict the Fermi gauge (recovered at $\mu_{\mathrm{IR}} \rightarrow 0$ ). Note also that in this case the ghosts contribute to the effective potential.

\section{THE STANDARD MODEL IN THE FERMI GAUGE}

It is a straightforward exercise to extend the results for the Abelian Higgs model, discussed in the previous sections, to the non-Abelian case and in particular to the SM. The (electroweak) gauge-fixing terms in the Lagrangian, in the Fermi gauge, are

$$
\mathcal{L}_{\mathrm{gf}}=-\frac{1}{2 \xi_{B}}\left(\partial^{\mu} B_{\mu}\right)^{2}-\frac{1}{2 \xi_{W}}\left(\partial^{\mu} W_{\mu}^{a}\right)^{2},
$$

where $B_{\mu}$ is now the $U(1)_{Y}$ gauge boson and $W_{\mu}^{a}$ are the $S U(2)_{L}$ ones. The Higgs doublet, with hypercharge $Y=1 / 2$, is written as

$$
H=\left(\begin{array}{c}
\chi^{+} \\
\frac{1}{\sqrt{2}}(h+i \chi)
\end{array}\right) \text {. }
$$

The potential is a function of the neutral field $h$ and the $\chi, \chi^{ \pm}$ fields are the three Goldstones.

The renormalized $\overline{\mathrm{MS}}$ effective potential, calculated up to one-loop order, has the form 


$$
V=-\frac{1}{2} m^{2} h^{2}+\frac{1}{4} \lambda h^{4}+\frac{\kappa}{4} \sum_{\alpha} N_{\alpha} M_{\alpha}^{4}(h)\left(\log \frac{M_{\alpha}^{2}(h)}{\mu^{2}}-C_{\alpha}\right)
$$

where $\alpha$ runs over all particle species, with $N_{\alpha}$ counting the corresponding degrees of freedom (taken negative for fermions) and tree-level mass-squared $M_{\alpha}^{2}(h)$ in the $h$ background. The $C_{\alpha}$ are constants (equal to $3 / 2$ for scalars and fermions, and to $5 / 6$ for the gauge bosons). The particle species and masses relevant for the potential are

$$
\begin{aligned}
& \text { Top quark: } N_{t}=-12, \quad T \equiv M_{t}^{2}=\frac{1}{2} y_{t}^{2} h^{2}, \\
& W^{ \pm}: N_{W}=6, \quad W \equiv M_{W}^{2}=\frac{1}{4} g^{2} h^{2}, \\
& Z^{0}: N_{Z}=3, \quad Z \equiv M_{Z}^{2}=\frac{1}{4}\left(g^{2}+g^{2}\right) h^{2}, \\
& \text { Higgs: } N_{h}=1, \quad H \equiv M_{h}^{2}=-m^{2}+3 \lambda h^{2}, \\
& \text { Charged Goldstones: } N_{A_{ \pm}}=2, \quad G_{A_{ \pm}} \equiv M_{A_{ \pm}}^{2}=\frac{1}{2}\left(G \pm \sqrt{G^{2}-4 \xi_{W} G W}\right) \text {, } \\
& \text { Neutral Goldstones: } N_{B_{ \pm}}=1, \quad G_{B_{ \pm}} \equiv M_{B_{ \pm}}^{2}=\frac{1}{2}\left[G \pm \sqrt{G^{2}-4\left(\xi_{W} W+\xi_{B} B\right) G}\right. \text {, }
\end{aligned}
$$

where we have used the auxiliary squared masses

$$
B \equiv \frac{1}{4} g^{2} h^{2}=Z-W, \quad G \equiv-m^{2}+\lambda h^{2} .
$$

As in the Abelian case, the minimum of the treelevel potential corresponds to $G=0$. The above expression for the effective potential is well known; see, e.g., $[19,33]$.

The IR properties of the potential in the limit $G \rightarrow 0$ are similar to those discussed in the Abelian model. There are IR divergences at higher orders in the perturbative expansion of the potential that can be resummed as in the Landau gauge [22,23], by the shift $G \rightarrow G+\Pi_{g}$, where now, at one-loop order,

$$
\begin{aligned}
\Pi_{g}= & \kappa\left[3 \lambda H\left(L_{H}-1\right)-6 y_{t}^{2} T\left(L_{T}-1\right)+\frac{3}{2} g^{2} W\left(L_{W}-1 / 3\right)\right. \\
& \left.+\frac{3}{4}\left(g^{2}+g^{\prime 2}\right) Z\left(L_{Z}-1 / 3\right)\right]
\end{aligned}
$$

where $L_{X}=\log \left(X / \mu^{2}\right)$. This resummation also solves the issue of residual gauge dependence at high field values in the region of instability [19], as discussed in subsection II C.

As in the Abelian case, however, this resummation still leaves a potential that suffers from an IR divergence in its first derivative. More concretely, the one-loop effective potential, expanded at small $G$ contains the terms

$$
\begin{aligned}
\delta V= & -\frac{\kappa}{4} G\left[2 \xi_{W} W \log \left(\frac{\xi_{W} G W}{\mu^{4}}\right)\right. \\
& \left.+\left(\xi_{W} W+\xi_{B} B\right) \log \left(\frac{\xi_{W} G W+\xi_{B} G B}{\mu^{4}}\right)\right],
\end{aligned}
$$

which are responsible for producing an IR divergence in $V^{\prime}$. There are no qualitative differences between this case and the Abelian one, so that again observable quantities like pole masses (for the Higgs boson and also for gauge bosons and fermions) are IR finite; IR divergences cancel out in the Nielsen identity; and the same kind of solutions discussed in Sec. IV can be applied to get rid of this complication and obtain an IR finite potential. In particular, the field redefinition that would achieve this is now

$$
h \rightarrow h+\frac{\kappa}{16} h\left(3 \xi_{W} g^{2}+\xi_{B} g^{2}\right) \log \left(\frac{G}{X}\right)
$$

where $X$ is left unspecified and can be chosen at will. For instance, the choice

$$
\log X=2\left(3 \xi_{W} W+\xi_{B} B\right) \log \mu^{2}
$$

modifies the one-loop anomalous dimension of the field and removes from it the $\xi_{W, Z}$ dependence, in the same way that this could be done in the Abelian model. If, instead, $X$ does not depend explicitly on $\mu$, the anomalous dimension is not modified.

The explicit expression of the one-loop potential after shifting the field as in Eq. (51) is 


$$
\begin{aligned}
V= & -\frac{1}{2} m^{2}+\frac{1}{4} \lambda h^{4}+\frac{\kappa}{4}\left[-12 T^{2}\left(L_{T}-3 / 2\right)+6 W^{2}\left(L_{W}-5 / 6\right)+3 Z^{2}\left(L_{Z}-5 / 6\right)+H^{2}\left(L_{H}-3 / 2\right)\right] \\
& +\frac{\kappa}{8} G\left[2 G\left(\log \frac{\xi_{W} G W}{\mu^{4}}-3\right)+G\left(\log \frac{G\left(\xi_{W} W+\xi_{B} B\right)}{\mu^{4}}-3\right)\right. \\
& -4 \xi_{W} W\left(\log \frac{\xi_{W} X W}{\mu^{4}}-3\right)-2\left(\xi_{W} W+\xi_{B} B\right)\left(\log \frac{X\left(\xi_{W} W+\xi_{B} B\right)}{\mu^{4}}-3\right) \\
& \left.+2\left(G_{A_{+}}-G_{A_{-}}\right) \log \frac{G_{A_{+}}}{G_{A_{-}}}+\left(G_{B_{+}}-G_{B_{-}}\right) \log \frac{G_{B_{+}}}{G_{B_{-}}}\right]
\end{aligned}
$$

Concerning the gauge dependence of the potential, it is similarly described by Nielsen identities of the form (23), one for each $\xi$ parameter (with a different $C$ function each) and the results discussed for the Abelian model carry over in a straightforward manner to the SM.

\section{CONCLUSIONS}

In some common gauges, like the Landau or Fermi gauge, Goldstone bosons are massless in the potential minimum in the broken phase and this causes IR divergences in the calculation of the perturbative effective potential. As demonstrated recently, these divergences are spurious and can be eliminated by a simple resummation of Goldstone self-energy diagrams that otherwise lead to the breakdown of perturbation theory $[18,22,23]$.

On the other hand, when one is dealing with a potential whose minimum is generated radiatively (and this includes not only the well-known Coleman-Weinberg model but also the SM potential at high field values) the value of the potential at that minimum (a gauge-independent quantity in principle) has a residual gauge dependence that also needs resummation of a tower of diagrams involving Goldstone bosons. In this paper, we have shown that the resummation of IR divergences mentioned previously automatically takes care of the residual gauge dependence in radiative minima.

We have shown this explicitly in the case of the Abelian Higgs model in the Fermi gauge, and in doing this we encountered a different IR problem: the first derivative of the potential (and therefore formally also the location of the minimum in a perturbative expansion) is IR divergent. This divergence can be traced back to a pole of order $p^{4}$ in the mixed propagator of the Goldstone bosons and longitudinal gauge bosons. As we showed, this pole is not an artifact of perturbation theory but a property of the full propagator. This mixed propagator is a specific feature of the Fermi gauge and so this IR divergence is not present in the Landau gauge $(\xi \rightarrow 0)$ nor in the background $R_{\xi}$ gauges.

Although naively this seems to be a serious pathology of the Fermi gauge, interestingly the IR divergence does not propagate to physical observables. We showed explicitly that all IR divergences cancel in the physical Higgs boson mass relation as well as in the Nielsen identity, which indicates that one can extract physical information from the effective potential in the Fermi gauge. Still, working with an effective potential with a spurious cusp seems odd. Our proposal to solve this issue (besides using an IR regulator to be removed at the end of the calculations) is to remove the IR divergence of the potential by an appropriate rescaling of the Higgs field, as described in Sec. IV. Several options for this rescaling are possible (with different advantages depending on the objective one has) and using any of them it is possible to have a well-behaved (IR finite) effective potential in the Fermi gauge. It could be argued that our field redefinitions either fix the gauge (if all $\xi$ dependence of the potential is removed by the redefinition) or amount to changing to a different gauge-fixing (some kind of interpolation between the Fermi and Landau gauges). In the latter case, we get the best of both worlds: we inherit the good IR properties of the Landau gauge, and we still have a free $\xi$ parameter to check gauge independence explicitly.

\section{ACKNOWLEDGMENTS}

We thank Enrico Bertuzzo, Joan Elias-Miró, Gian Giudice, Luca di Luzio, Antonio Riotto, Nikos Tetradis, and Alessandro Strumia for very useful discussions. J. R. E. thanks the CERN TH-Division for hospitality and partial financial support during several stages of this project. M. G. thanks the CPHT at the Ecole Polytechnique (Palaiseau, France) for hospitality and partial support during the final stage of this project. This work has been partly supported by the ERC Grant No. 669668-NEO-NAT-ERC-AdG-2014, the Spanish Ministry MINECO under Grants No. FPA201344773-P and No. FPA2014-55613-P, the Severo Ochoa excellence program of MINECO (Grant No. SEV-20120234) and by the Generalitat Grant No. 2014-SGR-1450. M. G. and T. K. acknowledge partial support by the Munich Institute for Astro- and Particle Physics (MIAPP) of the DFG cluster of excellence "Origin and Structure of the Universe." We also acknowledge support by the German Science Foundation (DFG) within the Collaborative Research Center (SFB) 676 Particles, Strings and the Early Universe.

\section{APPENDIX A: NONPERTURBATIVE PERSISTENCE OF THE IR PROBLEM}

In this appendix, we show that the IR problem of the Fermi gauge identified in the text persists 
nonperturbatively. In particular, we show that the full Goldstone propagator goes as $1 / k^{4}$ in the broken phase. The proof is based on the Ward-Takahashi identities, which we review first.

\section{BRS and Ward-Takahashi identities}

We shortly review the Ward-Takahashi identities in the Abelian Higgs model in the Fermi gauge. As usual, it is convenient to introduce an auxiliary field $B$ such that the gauge-fixing term reads

$$
\mathcal{L}_{G F}=F B+\frac{\xi}{2} B^{2}
$$

with $F=\partial_{\mu} B^{\mu}$ corresponding to the Fermi gauge. Solving for the equation of motion for $B$ gives $B=-F / \xi$, and replacing this in the gauge-fixing terms gives the usual expression.

The Lagrangian term involving Faddeev-Popov ghosts reads

$\mathcal{L}_{\mathrm{FP}}=-\bar{c}\left[\frac{\delta F}{\delta B_{\mu}} \partial_{\mu}+\frac{\delta F}{\delta \phi}(i g \phi)+\frac{\delta F}{\delta \phi^{*}}\left(-i g \phi^{*}\right)\right] c=-\bar{c} \square c$.

Under the BRS transformation the fields transform as

$$
\phi_{i} \rightarrow \phi_{i}+\theta \delta_{\mathrm{BRS}} \phi_{i}
$$

with a Grassmann parameter $\theta$ and where $\phi_{i}$ labels all fields (gauge, Higgs/Goldstone, auxiliary, ghost) with

$$
\begin{aligned}
\delta_{\mathrm{BRS}} A_{\mu} & =\partial_{\mu} c, \quad \delta_{\mathrm{BRS}} \phi=-i g \phi c, \\
\delta_{\mathrm{BRS}} c & =0, \quad \delta_{\mathrm{BRS}} \bar{c}=B, \quad \delta_{\mathrm{BRS}} B=0 .
\end{aligned}
$$

It is convenient to split the Higgs field in components as $\phi=(v+h+i \chi) / \sqrt{2}$. Their BRS transformation is then

$$
\delta_{\mathrm{BRS}} h=g \chi c, \quad \delta_{\mathrm{BRS}} \chi=-g(v+h) c .
$$

The generating functional in the presence of sources $J$ and $K$ reads

$$
e^{i W[J, K]} \equiv\left\langle 0\left|T e^{i \int d^{d} x\left(J_{i}(x) \phi_{i}(x)+K_{i}(x) \delta_{\mathrm{BRS}} \phi_{i}(x)\right.}\right| 0\right\rangle,
$$

where $K$ sources the BRS transformation. The expectation value of the fields can then be written as

$$
\phi_{i}(x)=\frac{\delta W}{\delta J_{i}(x)}
$$

and the effective action is obtained via a Legendre transformation [we use the short-hand notation $J_{i} \phi_{i}=$ $\left.\int d^{d} x J_{i}(x) \phi_{i}(x)\right]$

$$
\Gamma[\phi, K]=W[J[\phi, K], K]-J_{i}[\phi, K] \phi_{i},
$$

Under the BRS transformations the energy functional $W$ behaves as

$$
W[J, K] \rightarrow W[J, K+\lambda J] \Rightarrow \delta_{\mathrm{BRS}} W=J_{i} \frac{\delta W}{\delta K_{i}},
$$

where we used that the BRS transformation is nilpotent, namely $\delta_{\mathrm{BRS}}\left(\delta_{\mathrm{BRS}} \phi_{i}\right)=0$. At the same time, since the BRS transformation can be absorbed into the integration measure, one finds $\delta_{\mathrm{BRS}} W=0$. Using

$$
\left.\frac{\delta W}{\delta K}\right|_{J=\mathrm{const}}=\left.\frac{\delta \Gamma}{\delta K}\right|_{\phi=\mathrm{const}}, \quad J_{i}=-\frac{\delta \Gamma}{\delta \phi_{i}},
$$

one obtains

$0=\frac{\delta \Gamma}{\delta \phi_{i}} \frac{\delta \Gamma}{\delta K_{i}}=\frac{\delta \Gamma}{\delta B_{\mu}} \frac{\delta \Gamma}{\delta K^{\mu}}+\frac{\delta \Gamma}{\delta h} \frac{\delta \Gamma}{\delta K^{h}}+\frac{\delta \Gamma}{\delta \chi} \frac{\delta \Gamma}{\delta K^{\chi}}+\frac{\delta \Gamma}{\delta \bar{c}} \frac{\delta \Gamma}{\delta K^{\bar{c}}}$.

In the last expression we used already that the BRS transformation of $c$ and $B$ vanishes, so that the effective action is independent of the corresponding sources $K^{B}$ and $K^{c}$.

\section{Ward identities for the gauge boson propagator}

Let us next derive the Ward identities for the gauge boson propagator. In the following we assume that $v$ is the full vacuum expectation value, i.e., that $\langle h\rangle=v$ is a solution of the equations of motion for vanishing external source. Furthermore, the symmetry $\chi \rightarrow-\chi, B_{\mu} \rightarrow-B_{\mu}$ guarantees that $\langle\chi\rangle=\left\langle B_{\mu}\right\rangle=0$ is a solution of the equations of motion. This is equivalent to the condition that the Fermi gauge is a "good gauge" in the sense of Fukuda-Kugo [34]. Finally, the symmetry $c \rightarrow-c, \bar{c} \rightarrow-\bar{c}$ guarantees that the vanishing ghost expectation value is always a solution to the equations of motion; i.e., it corresponds to a configuration with vanishing external sources.

The full inverse propagator for the gauge field, the Goldstone boson, and their mixing is given by

$$
\mathcal{G}^{-1}(x, y)=-i\left(\begin{array}{cc}
\frac{\delta^{2} \Gamma}{\delta B_{\nu} \delta B_{\mu}} & \frac{\delta^{2} \Gamma}{\delta B_{\nu} \delta \chi} \\
\frac{\delta^{2} \Gamma}{\delta \chi \delta B_{\mu}} & \frac{\delta^{2} \Gamma}{\delta \chi \delta \chi}
\end{array}\right)
$$

where $\Gamma$ is the effective action. One can obtain a WT identity for this propagator by taking derivatives of (A11) with respect to $B_{\nu}$ and $\chi$. In addition, we take a derivative with respect to the ghost field $c$, and then set all expectation values to zero except for the Higgs, which is assumed to be in the broken minimum. As discussed above, since the 
Fermi gauge is a "good gauge," this corresponds to a solution of the equations of motion; i.e., all sources vanish. The symmetry $\chi \rightarrow-\chi, B_{\mu} \rightarrow-B_{\mu}$ implies that second derivatives involving one Higgs and one Goldstone or gauge field, vanish. Furthermore, all first derivatives of $\Gamma$ with respect to any field vanish due to the on-shell stationarity of the effective action. Finally, using that $\delta \Gamma / \delta K_{i}=\delta W / \delta K_{i}=\left\langle\delta_{\mathrm{BRS}} \phi_{i}\right\rangle$, and using the BRS transformations as well as ghost number conservation, it follows that most contributions are zero for vanishing (ghost) background field, except terms involving $\delta^{2} \Gamma /\left(\delta K_{i} \delta c\right)$ for $i=h, \chi, B_{\mu}$ and terms involving $\delta^{2} \Gamma /(\delta c \delta \bar{c})$. Writing the result in matrix form, one obtains

$$
\mathcal{G}^{-1}\left(\begin{array}{c}
\frac{\delta^{2} \Gamma}{\delta K^{\mu} \delta c} \\
\frac{\delta^{2} \Gamma}{\delta K^{\chi} \delta c}
\end{array}\right)+(-i) \frac{\delta^{2} \Gamma}{\delta c \delta \bar{c}}\left(\begin{array}{c}
\frac{\delta B}{\delta B_{\nu}} \\
\frac{\delta B}{\delta \chi}
\end{array}\right)=0 .
$$

Here we have used already that $\delta \Gamma / \delta K^{\bar{c}}=\left\langle\delta_{\mathrm{BRS}} \bar{c}\right\rangle=$ $\langle\hat{B}\rangle=B$, where we have denoted the field operator by a hat here and assumed in the last equality that the auxiliary field is linear in the fundamental fields, i.e., that the gaugefixing function $F$ is a linear function of the field variables.

Specifying to the Fermi gauge where $B=-F / \xi=$ $-\partial_{\mu} B^{\mu} / \xi$ and the ghost is a free field [such that its inverse propagator is simply $\delta^{2} \Gamma /(\delta c \delta \bar{c})=\delta^{2} S /(\delta c \delta \bar{c})=k^{2}$, one obtains in Fourier space:

$$
\mathcal{G}^{-1}(k)\left(\begin{array}{c}
\frac{\delta^{2} \Gamma}{\delta K^{\mu} \delta c} \\
\frac{\delta^{2} \Gamma}{\delta K^{\chi} \delta c}
\end{array}\right)-k^{2}\left(\begin{array}{c}
k_{\nu} / \xi \\
0
\end{array}\right)=0 .
$$

One has $\delta^{2} \Gamma /\left[\delta K^{\mu}(y) \delta c(z)\right]=\delta\left[\left\langle\delta_{\mathrm{BRS}} B^{\mu}(y)\right\rangle\right] / \delta c(z)=$ $\delta\left[\partial^{\mu} c(y)\right] / \delta c(z)=\partial^{\mu} \delta(y-z)$. Since the ghost is a free field one also gets $\delta^{2} \Gamma /\left[\delta K^{\chi}(y) \delta c(z)\right]=\delta[\langle(-g(v+$ $\hat{h}(y)) \hat{c}(y)\rangle] / \delta c(z)=\delta[\langle(-g(v+\hat{h}(y))\rangle\langle\hat{c}(y)\rangle] / \delta c(z)=$ $-g v \delta(y-z)$. In the last step we used $\langle h\rangle=0$, i.e., the assumption that one expands around the position of the broken minimum. In Fourier space, this gives

$$
\mathcal{G}^{-1}(k)\left(\begin{array}{c}
i k^{\mu} \\
g v
\end{array}\right)+k^{2}\left(\begin{array}{c}
k_{\nu} / \xi \\
0
\end{array}\right)=0 \text {. }
$$

It is easy to check that the WT identity is fulfilled at tree level, where

$\mathcal{G}_{0}^{-1}(k)=-i\left(\begin{array}{cc}-g_{\mu \nu} k^{2}+k_{\mu} k_{\nu}-k_{\mu} k_{\nu} / \xi+g^{2} v^{2} g_{\mu \nu} & -i k_{\nu} g v \\ i k_{\mu} g v & k^{2}-m_{\chi}^{2}\end{array}\right)$,

and $m_{\chi}^{2}=0$ in the broken phase.

To see how the $k^{4}$ pole arises in general, we can decompose the propagator into transverse and longitudinal parts (using the short-hand notation $\hat{k}_{\mu} \equiv k_{\mu} / \sqrt{k^{2}} \equiv k_{\mu} / k$ ) as

$$
\begin{aligned}
\mathcal{G}(k)= & \left(\begin{array}{cc}
g_{\mu \nu}-\hat{k}_{\mu} \hat{k}_{\nu} & 0 \\
0 & 0
\end{array}\right) \mathcal{G}_{T}(k) \\
& +\left(\begin{array}{cc}
-i \hat{k}_{\mu} & 0 \\
0 & 1
\end{array}\right) \mathcal{G}_{L}(k)\left(\begin{array}{cc}
i \hat{k}_{\nu} & 0 \\
0 & 1
\end{array}\right),
\end{aligned}
$$

where $\mathcal{G}_{T}(k)$ is a function but $\mathcal{G}_{L}(k)$ still has a $2 \times 2$ matrix structure and both are Lorentz scalars. The above WT identity then takes the form

$$
\mathcal{G}_{L}^{-1}(k)\left(\begin{array}{c}
k \\
g v
\end{array}\right)-i \frac{k^{3}}{\xi}\left(\begin{array}{l}
1 \\
0
\end{array}\right)=0
$$

Using the fact that the Goldstone mass vanishes in the broken phase, the longitudinal propagator must have the form

$\mathcal{G}_{L}^{-1}(k)=\frac{i}{\xi} k^{2}\left(\begin{array}{ll}1 & 0 \\ 0 & 0\end{array}\right)-i A(k)\left(\begin{array}{cc}g^{2} v^{2} & -k g v \\ -k g v & k^{2}\end{array}\right)$,

with some function $A(k)$ that has no poles at $k^{2}=0$. This form means that $\mathcal{G}_{L}$ has a pole that goes (at least) like $1 / k^{4}$, as $\operatorname{det} \mathcal{G}_{L}=\xi /\left[k^{4} A(k)\right]$.

Another way of understanding Eq. (A19) is in terms of operators. The WT equation states that the operator $\left|D_{\mu} \phi\right|^{2}$ that induces the second contribution in (A19) is radiatively corrected while the gauge-breaking term is not. The running of the parameter $\xi$, hence, stems from wavefunction renormalization of the gauge fields. We explicitly confirmed this at the one-loop level.

\section{APPENDIX B: HIGGS SELF-ENERGY AND CORRECTION TO THE KINETIC TERM}

In this appendix, we present the full self-energy $\Sigma$ of the physical Higgs at one loop in the Abelian Higgs model. We separate $\Sigma$ in different pieces as

$\Sigma=\Sigma_{L L}+2 \Sigma_{L T}+\Sigma_{T T}+\Sigma_{H H}+\Sigma_{L}+\Sigma_{T}+\Sigma_{H}$,

where the indices denote the type of fields propagating in the loop (one index for loops with a quartic vertex and two indices for loops with cubic vertices). Here, $L$ labels the mixed longitudinal and Goldstone fields, $T$ the transverse part of the gauge field, and $H$ the physical Higgs. We find [with the squared masses $H, G$ and $B$ as defined in (3)] 


$$
\begin{aligned}
& \Sigma_{H H}\left(p^{2}\right)=i \mu^{4-d} 18 \lambda^{2} h^{2} \int \frac{d^{d} k}{(2 \pi)^{d}} \frac{1}{\left(k_{-}^{2}-H\right)\left(k_{+}^{2}-H\right)}, \\
& \Sigma_{T T}\left(p^{2}\right)=i \mu^{4-d} \frac{2 B^{2}}{h^{2}} \int \frac{d^{d} k}{(2 \pi)^{d}} \frac{1}{\left(k_{-}^{2}-B\right)\left(k_{+}^{2}-B\right)}\left[d-2+\frac{\left(k_{+}^{2}+k_{-}^{2}-p^{2}\right)^{2}}{4 k_{-}^{2} k_{+}^{2}}\right], \\
& \Sigma_{L T}\left(p^{2}\right)=i \mu^{4-d} \frac{B}{2 h^{2}} \int \frac{d^{d} k}{(2 \pi)^{d}} \frac{p^{2}\left(p^{2}-4 k_{-}^{2}\right)\left(k_{+}^{4}+B G \xi\right)}{k_{-}^{2} k_{+}^{2}\left(k_{-}^{2}-B\right)\left(k_{+}^{4}-G k_{+}^{2}+B G \xi\right)}, \\
& \Sigma_{L L}\left(p^{2}\right)=i \mu^{4-d} \int \frac{d^{d} k}{(2 \pi)^{d}} \frac{1}{\left(k_{-}^{4}-G k_{-}^{2}+B G \xi\right)\left(k_{+}^{4}-G k_{+}^{2}+B G \xi\right)} \sum_{i, j=1}^{5} E_{i j} k_{-}^{2 i-4} k_{+}^{2 j-4},
\end{aligned}
$$

where the $E_{i j}$ entering $\Sigma_{L L}\left(p^{2}\right)$ is given by the matrix

$$
E \equiv\left(\begin{array}{ccccc}
B^{2} G^{2} p^{4} \xi^{2} & -2 B^{2} G^{2} p^{2} \xi^{2} & B G \xi\left(p^{4}+B G \xi\right) & -2 B G p^{2} \xi & B G \xi \\
-2 B^{2} G^{2} p^{2} \xi^{2} & B^{2}\left(2 G^{2}+\Delta^{4}\right) \xi^{2} & -B \xi\left(\Delta^{4}+B G \xi\right) & 2 B \Delta^{2} \xi & -B \xi \\
B G \xi\left(p^{4}+B G \xi\right) & -B \xi\left(\Delta^{4}+B G \xi\right) & 4 \lambda^{2} h^{4} & 0 & 0 \\
-2 B G p^{2} \xi & 2 B \Delta^{2} \xi & 0 & 0 & 0 \\
B G \xi & -B \xi & 0 & 0 & 0
\end{array}\right),
$$

with $\Delta^{2} \equiv p^{2}-2 \lambda h^{2}, k_{ \pm}^{2} \equiv(k \pm p / 2)^{2}$, and

$$
\begin{aligned}
\Sigma_{H} & =3 i \lambda \mu^{4-d} \int \frac{d^{d} k}{(2 \pi)^{d}} \frac{1}{k^{2}-H}, \\
\Sigma_{T} & =i \frac{(d-1) B}{h^{2}} \mu^{4-d} \int \frac{d^{d} k}{(2 \pi)^{d}} \frac{1}{k^{2}-B}, \\
\Sigma_{L} & =i \mu^{4-d} \int \frac{d^{d} k}{(2 \pi)^{d}} \frac{k^{2}\left(\lambda h^{2}+\xi B\right)-\xi B\left(\lambda h^{2}+G\right)}{h^{2}\left(k^{4}-G k^{2}+B G \xi\right)} .
\end{aligned}
$$

All loop integrals can be reduced to the elementary oneloop functions,

$$
\begin{gathered}
A(X) \equiv-i \mu^{4-d} 16 \pi^{2} \int \frac{d^{d} k}{(2 \pi)^{d}} \frac{1}{k^{2}-X} \\
=m^{2}\left[\Delta_{\epsilon}+1-\log \left(X / \mu^{2}\right)\right], \\
B\left(X_{1}, X_{2}, p^{2}\right) \\
\equiv-i \mu^{4-d} 16 \pi^{2} \int \frac{d^{d} k}{(2 \pi)^{d}} \frac{1}{\left(k_{-}^{2}-X_{1}-i \varepsilon\right)} \frac{1}{\left(k_{+}^{2}-X_{2}-i \varepsilon\right)} \\
=\Delta_{\epsilon}-\int_{0}^{1} d x \log \left[\frac{X_{1}(1-x)+X_{2} x-x(1-x) p^{2}-i \varepsilon}{\mu^{2}}\right],
\end{gathered}
$$

with $\Delta_{\epsilon} \equiv 2 /(4-d)-\gamma_{E}+\log 4 \pi$. After factorizing,

$$
N(k) \equiv k^{4}-G k^{2}+B G \xi=\left(k^{2}-G_{-}^{2}\right)\left(k^{2}-G_{+}^{2}\right),
$$

with $G_{ \pm}$as defined in (6), the reduction can be done in several ways. For example, in $\Sigma_{T T}$ one can write the numerator of the last term as

$$
\begin{aligned}
\left(k_{+}^{2}+k_{-}^{2}-p^{2}\right)^{2}= & 2 k_{+}^{2} k_{-}^{2}+p^{4}+k_{+}^{2}\left(k_{+}^{2}-B\right) \\
& +k_{-}^{2}\left(k_{-}^{2}-B\right)+\left(B-2 p^{2}\right)\left(k_{+}^{2}+k_{-}^{2}\right),
\end{aligned}
$$

and then split the integral into contributions from each summand. Similarly, in $\Sigma_{L T}$, one can add and subtract $G k_{+}^{2}$ in the right-most bracket of the numerator, and in $\Sigma_{L}$ one can write $k^{2}=\left[\left(k^{2}-G_{-}\right)+\left(k^{2}-G_{+}\right)\right] / 2+G / 2$. One then finds

$$
\begin{aligned}
\Sigma_{H H}\left(p^{2}\right)= & -18 \kappa \lambda^{2} h^{2} B\left(H, H, p^{2}\right), \\
\Sigma_{T T}\left(p^{2}\right)= & -\frac{2 \kappa B^{2}}{v^{2}}\left[B\left(T, T, p^{2}\right)(d-3 / 2)\right. \\
& +\frac{\left(B-2 p^{2}\right)}{2} B^{2,1}\left(0, B, B, p^{2}\right) \\
& \left.+\frac{1}{2} B(0, B, 0)+\frac{p^{4}}{4} B^{2,2}\left(0, B, 0, B, p^{2}\right)\right], \\
\Sigma_{L T}\left(p^{2}\right)= & -\kappa \frac{p^{2} B}{2 h^{2}}\left[p^{2} B^{2,1}\left(0, B, 0, p^{2}\right)-4 B\left(0, B, p^{2}\right)\right. \\
& +G p^{2} B^{2,2}\left(0, B, G_{+}, G_{-}, p^{2}\right) \\
& \left.-4 G B^{2,1}\left(G_{+}, G_{-}, B, p^{2}\right)\right],
\end{aligned}
$$

and 


$$
\begin{aligned}
& \Sigma_{H}=-3 \kappa \lambda H A(H), \\
& \Sigma_{T}=-\kappa \frac{B^{2}}{h^{2}}(d-1) A(B), \\
& \Sigma_{L}=-\kappa \frac{\left(G \lambda h^{2}-\xi B G-2 \xi B \lambda h^{2}\right)}{2 h^{2}} B\left(G_{+}, G_{-}, 0\right)-\frac{\kappa}{2 h^{2}}\left[A\left(G_{+}\right)+A\left(G_{+}\right)\right],
\end{aligned}
$$

where $\kappa=1 /\left(16 \pi^{2}\right)$ and

$$
\begin{aligned}
B^{2,1}\left(X_{1}, X_{2}, X_{3}, p^{2}\right) & \equiv-i \mu^{4-d} 16 \pi^{2} \int \frac{d^{d} k}{(2 \pi)^{d}} \frac{1}{\left(k_{-}^{2}-X_{1}-i \varepsilon\right)} \frac{1}{\left(k_{-}^{2}-X_{2}-i \varepsilon\right)} \frac{1}{\left(k_{+}^{2}-X_{3}-i \varepsilon\right)} \\
& =\frac{1}{X_{1}-X_{2}}\left[B\left(X_{1}, X_{3}, p^{2}\right)-B\left(X_{2}, X_{3}, p^{2}\right)\right] .
\end{aligned}
$$

Similarly, $B^{2,2}$ contains two propagators involving $k_{-}^{2}$ and $k_{+}^{2}$, respectively, and is

$$
B^{2,2}\left(X_{1}, X_{2}, X_{3}, X_{4}, p^{2}\right)=\frac{B\left(X_{1}, X_{3}, p^{2}\right)-B\left(X_{1}, X_{4}, p^{2}\right)-B\left(X_{2}, X_{3}, p^{2}\right)+B\left(X_{2}, X_{4}, p^{2}\right)}{\left(X_{1}-X_{2}\right)\left(X_{3}-X_{4}\right)} .
$$

The piece $\Sigma_{L L}$ can be reduced by first rewriting the powers of $k_{ \pm}^{2}$ for $i=4,5$ or $j=4,5$ as

$$
\begin{aligned}
& k_{ \pm}^{4}=N\left(k_{ \pm}\right)+G k_{ \pm}^{2}-B G \xi \\
& k_{ \pm}^{6}=\left(k_{ \pm}^{2}+G\right) N\left(k_{ \pm}\right)+G(G-B \xi) k_{ \pm}^{2}-B G^{2} \xi
\end{aligned}
$$

Then one obtains integrals that can be reduced as above, for example,

$$
\begin{aligned}
-i \mu^{4-d} 16 \pi^{2} \int \frac{d^{d} k}{(2 \pi)^{d}} \frac{k_{-}^{2}}{N\left(k_{+}\right) N\left(k_{-}\right)}= & \frac{1}{2}\left[B^{2,1}\left(G_{+}, G_{-}, G_{+}, p^{2}\right)+B^{2,1}\left(G_{+}, G_{-}, G_{-}, p^{2}\right)\right. \\
& \left.+G B^{2,2}\left(G_{+}, G_{-}, G_{+}, G_{-}, p^{2}\right)\right]
\end{aligned}
$$

where we rewrote $k_{-}^{2}$ in the numerator as done for $\Sigma_{L}$. Another useful relation is

$$
\int \frac{d^{d} k}{(2 \pi)^{d}} \frac{k_{-}^{2}}{N\left(k_{+}\right)}=\int \frac{d^{d} k}{(2 \pi)^{d}} \frac{k^{2}+p^{2}}{N(k)}
$$

The full result for $\Sigma_{L L}$ is straightforward but too lengthy to report. A useful check is that $\Sigma\left(p^{2}=0\right)=V_{1}^{\prime \prime}$. To get the self-energy at finite $p^{2}$ in the limit $G \rightarrow 0$, we used the expansion

$$
\begin{aligned}
\int_{0}^{1} d x \log [a(1-x)+b x-x(1-x) c]= & -2+\frac{1}{2} \log (a b)+\frac{a-b}{2 c} \log \left(\frac{a}{b}\right)+\frac{S}{2 c} \log \left(\frac{c-a-b+S}{c-a-b-S}\right) \\
= & -2+\log (-c)+\frac{1}{c}[a(\log a-1)+b(\log b-1)-(a+b) \log (-c)] \\
& -\frac{1}{2 c^{2}}\left\{a^{2}+b^{2}+2 a b[\log (a b)-2 \log (-c)]\right\}+\mathcal{O}\left(\frac{1}{c^{3}} \log c\right)
\end{aligned}
$$

for small $a, b$, where $S=\sqrt{(c-a-b)^{2}-4 a b}$.

From the self-energies, one can get the corrections to the kinetic term in the effective action

$$
Z(h)=1-\left.\frac{d \Sigma\left(p^{2}\right)}{d p^{2}}\right|_{p^{2}=0}
$$


We find

$$
Z=1+Z_{L L}+2 Z_{L T}+Z_{T T}+Z_{H H}
$$

with the explicit results (for the Abelian Higgs model),

$$
\begin{aligned}
Z_{L L}= & \kappa \frac{\xi B}{h^{2}} \Delta_{\epsilon}+\frac{\kappa}{3 \pi^{2} h^{2}}\left\{G-3 \xi B\left[\frac{G_{+}\left(L_{G_{+}}+1\right)-G_{-}\left(L_{G_{-}}+1\right)}{\Delta G_{ \pm}}\right]+\frac{m^{2}}{\Delta G_{ \pm}^{4}}\left[\left(2 \Delta G_{ \pm}^{2}+m^{2} G\right)(G-6 \xi B)(G-\xi B)\right.\right. \\
& \left.\left.+6 \xi^{2} B^{2} G\left(\Delta G_{ \pm}^{2}+2 m^{2} \xi B\right) \frac{\left(L_{G_{+}}-L_{G_{-}}\right)}{\Delta G_{ \pm}}\right]\right\}, \\
& 2 Z_{L T}=-\frac{3 \kappa B}{h^{2}} \Delta_{\epsilon}+\frac{3 \kappa B}{2 h^{2} B_{\xi}}\left[2(B+\xi G)\left(L_{B}-\frac{5}{6}\right)-G\left(L_{G_{+}}+L_{G_{-}}-\frac{5}{3}\right)-G^{2}(1-2 \xi)\left(\frac{L_{G_{+}}-L_{G_{-}}}{\Delta G_{ \pm}}\right)\right],
\end{aligned}
$$

and

$$
Z_{T T}=\frac{5 \kappa B}{2 h^{2}}, \quad Z_{H H}=\frac{3 \kappa \lambda^{2} h^{2}}{H},
$$

where $L_{G_{ \pm}} \equiv \log \left(G_{ \pm} / \mu^{2}\right), L_{B} \equiv \log \left(B / \mu^{2}\right), B_{\xi} \equiv B+G(\xi-1)$ and $\Delta G_{ \pm} \equiv G_{+}-G_{-}$. For large field values one can approximate $G=-m^{2}+\lambda h^{2} \rightarrow \lambda h^{2}$ and neglect the second and third line in (B19) which are proportional to the quadratic mass parameter $m^{2}$. In that limit, $Z_{H H} \rightarrow \lambda \kappa$.

For completeness, we also present the off-shell Higgs self-energy for $h \rightarrow v$

$$
\begin{aligned}
\left.\Sigma\left(p^{2}\right)\right|_{h \rightarrow v}= & \frac{\kappa v^{2}}{2}\left\{2\left[-26 \lambda^{2}+5 \lambda g^{2} \xi+g^{2}(3-\xi) P^{2}-9 g^{4}\right] \frac{1}{\epsilon}+2 P^{4}\right. \\
& +\left[\log \left(p^{2} / \mu^{2}\right)+i \pi\right]\left(4 \lambda^{2}-P^{4}\right)+\xi g^{2}\left(P^{2}-5 \lambda\right) \log \left(\xi G B / \mu^{4}\right) \\
& +\lambda^{2}\left[-20+12 L_{H}+36 \int_{0}^{1} d x \log \left[\left(H-x(1-x) p^{2}\right) / \mu^{2}\right]\right] \\
& +6 g^{4}\left(1+L_{B}\right)+2 g^{2} P^{2}\left(1-\xi-L_{B}\right)+10 \lambda g^{2} \xi \\
& +\left(P^{4}-4 P^{2} g^{2}+12 g^{4}\right) \int_{0}^{1} d x \log \left[\left(B-x(1-x) p^{2}\right) / \mu^{2}\right] \\
& \left.-\xi g^{2} \frac{\left(P^{2}-2 \lambda\right)^{2}}{P^{2}}\left[\left(1-\frac{g^{2} \xi}{P^{2}}\right)\left[\log \left(\xi G B / p^{4}\right)-2 i \pi\right]-2\right]\right\}
\end{aligned}
$$

where $P^{2} \equiv p^{2} / v^{2}$.

\section{APPENDIX C: NIELSEN IDENTITY FOR THE KINETIC TERM}

The Nielsen identity for the kinetic term $\delta \mathcal{L}_{K}=Z(h)\left(\partial_{\mu} h\right)^{2} / 2 \operatorname{reads}[15,18]$

$$
\xi \frac{\partial Z}{\partial \xi}=-C \frac{\partial Z}{\partial h}-2 Z \frac{\partial C}{\partial h}+D \frac{\partial V}{\partial h}+\tilde{D} \frac{\partial^{2} V}{\partial h^{2}},
$$

with coefficients given by a gradient expansion of (24),

$$
C \rightarrow C+D\left(\partial_{\mu} h\right)^{2}-\partial_{\mu}\left(\tilde{D} \partial^{\mu} h\right)+\mathcal{O}\left(\partial^{4}\right)
$$

Note that Ref. [15] did not include the total derivative term above, which is relevant as it is required to describe the full $\xi$ dependence of the function $Z$.

At one loop, the contribution at zeroth order in gradients is given by the one-loop expressions of $C, D$, and $\tilde{D}$. The $C_{1}$ function is given in Eq. (25), and $D_{1}, \tilde{D}_{1}$ are 


$$
\begin{aligned}
\tilde{D}_{1}= & -\frac{\kappa G \xi}{3}\left[\frac{2 g^{2}}{G^{2}}+\frac{2(\xi B-G) B}{\Delta G_{ \pm}^{4}}\left(\lambda-4 g^{2} \xi\right)-3 B \xi\left[g^{2} G^{2}+2 \lambda B(2 \xi B-G)\right] \frac{\log \left(G_{+} / G_{-}\right)}{\Delta G_{ \pm}^{5}}\right] \\
D_{1}= & \frac{\kappa \xi G}{6 g^{2} h B_{\xi}^{2}}\left\{9 g^{4} \log \left(\frac{G \xi}{B}\right)+\frac{2 B_{\xi}}{\Delta G_{ \pm}^{6}}\left[-\lambda^{2} B^{2} B_{\xi}\left(3 G^{2}-4 G B \xi+28 B^{2} \xi^{2}\right)\right.\right. \\
& +g^{4} G^{2}\left[3 G^{3}(-4+\xi)+212 B^{3} \xi^{2}+G^{2} B\left(21+80 \xi-8 \xi^{2}\right)-4 G B^{2} \xi\left(38+17 \xi+19 \xi^{2}\right)\right] \\
& \left.-8 g^{2} \lambda \xi B^{2} B_{\xi}(G-B \xi)(G-24 B \xi)\right] \\
& -3 \frac{\log \left(G_{+} / G_{-}\right)}{\Delta G_{ \pm}^{7}}\left[g ^ { 4 } \left[\frac{3 G}{2}(2 \xi-1) \Delta G_{ \pm}^{4}\left[3 \Delta G_{ \pm}^{2}-G^{2}(-1+2 \xi)^{2}\right]\right.\right. \\
& \left.+\frac{B_{\xi}^{2}}{4 G}\left[-\Delta G_{ \pm}^{6}+G^{2} \Delta G_{ \pm}^{4}(13+48 \xi)+17 G^{4} \Delta G_{ \pm}^{2}-5 G^{6}\right]\right] \\
& \left.\left.-4 \xi \lambda B^{2} B_{\xi}^{2}\left[2 \lambda B\left(G^{2}-3 G B \xi+6 B^{2} \xi^{2}\right)+g^{2} G\left(G^{2}-16 G B \xi+8 B^{2} \xi^{2}\right)\right]\right]\right\}
\end{aligned}
$$

where $B_{\xi} \equiv B+G(\xi-1)$ and $\Delta G_{ \pm} \equiv G_{+}-G_{-}$. The small- $G$ expansion of these functions reads

$$
\begin{aligned}
& \tilde{D}_{1}=-\frac{\kappa \pi \lambda g h \xi^{1 / 2}}{8 G^{3 / 2}}-\frac{\kappa\left(\lambda+3 g^{2} \xi\right)}{6 G}-\frac{\kappa \lambda \pi}{64(B G \xi)^{1 / 2}}+\mathcal{O}\left(G^{0}\right), \\
& D_{1}=\frac{3 \kappa \pi \lambda^{2} g h^{2} \xi^{1 / 2}}{16 G^{5 / 2}}+\frac{\kappa \lambda h\left(\lambda+3 g^{2} \xi\right)}{3 G^{2}}+\frac{\kappa \pi \lambda\left(9 \lambda+16 g^{2} \xi\right)}{128 g \xi^{1 / 2} G^{3 / 2}}+\mathcal{O}\left(G^{-1}\right) .
\end{aligned}
$$

With these expressions, one can check explicitly that the Nielsen identity for $Z$ is fulfilled perturbatively at one loop.

\section{APPENDIX D: KINETIC TERM FOR THE SM IN THE FERMI GAUGE}

In the Standard Model, the kinetic term is readily obtained from the corresponding expressions in the Abelian Higgs model, given in Appendix B. We find

$$
Z=1+Z_{L L}+2 Z_{L^{+} L^{-}}+2 Z_{L T}+4 Z_{L^{+} T^{-}}+Z_{T T}+2 Z_{T^{+} T^{-}}+Z_{H H}+Z_{t t}
$$

with

$$
\begin{aligned}
Z_{H H} & =Z_{H H}^{U(1)}, \quad Z_{T T}=\left.Z_{T T}^{U(1)}\right|_{B \rightarrow Z}, \\
Z_{T^{+} T^{-}} & =\left.Z_{T T}^{U(1)}\right|_{B \rightarrow W}, \quad Z_{L T}=\left.Z_{L T}^{U(1)}\right|_{B \rightarrow Z, \xi \rightarrow \xi_{\text {eff }}}, \\
Z_{L^{+} T^{-}} & =\left.Z_{L T}^{U(1)}\right|_{B \rightarrow W, \xi \rightarrow \xi_{W}}, \quad Z_{L L}=\left.Z_{L L}^{U(1)}\right|_{B \rightarrow Z, \xi \rightarrow \xi_{\text {eff }}}, \\
Z_{L^{+} L^{-}} & =\left.Z_{L L}^{U(1)}\right|_{B \rightarrow W, \xi \rightarrow \xi_{W}}, \quad Z_{t t}=\frac{6 \kappa T}{h^{2}} \Delta_{\epsilon}-\frac{6 \kappa T}{h^{2}}\left[1 / 4+\log \left(T / \mu^{2}\right)\right],
\end{aligned}
$$

where $W=g^{2} h^{2} / 4, Z=\left(g^{2}+g^{2}\right) h^{2} / 4, B=Z-W, T=y_{t}^{2} h^{2} / 2$ and $\xi_{\text {eff }} \equiv\left(\xi_{B} B+\xi_{W} W\right) /(B+W)=\xi_{B} s_{W}^{2}+\xi_{W} c_{W}^{2}$. The divergent part is given by (note that the TT and hh parts are UV finite)

$$
\begin{aligned}
Z^{\mathrm{div}} & =\frac{\kappa}{h^{2}}\left(\xi_{\mathrm{eff}} Z+2 \xi_{W} W-3 Z-6 W+6 T\right) \Delta_{\epsilon} \\
& =\frac{\kappa}{4}\left(\xi_{B} g^{\prime 2}+3 \xi_{W} g^{2}-3 g^{\prime 2}-9 g^{2}+12 y_{t}^{2}\right) \Delta_{\epsilon} .
\end{aligned}
$$

This is consistent with Eq. (2.47) of [33]. In particular, the field renormalization $Z_{h}$ cancels the divergences in $Z$. 
[1] G. Aad et al. (ATLAS Collaboration), Phys. Lett. B 716, 1 (2012); S. Chatrchyan et al. (CMS Collaboration), Phys. Lett. B 716, 30 (2012).

[2] G. Aad et al. (ATLAS and CMS Collaborations), Phys. Rev. Lett. 114, 191803 (2015).

[3] ATLAS and CDF and CMS and D0 Collaborations, arXiv:1403.4427.

[4] G. Degrassi, S. Di Vita, J. Elias-Miró, J. R. Espinosa, G. F. Giudice, G. Isidori, and A. Strumia, J. High Energy Phys. 08 (2012) 098.

[5] D. Buttazzo, G. Degrassi, P. P. Giardino, G. F. Giudice, F. Sala, A. Salvio, and A. Strumia, J. High Energy Phys. 12 (2013) 089.

[6] F. Bezrukov, M. Y. Kalmykov, B. A. Kniehl, and M. Shaposhnikov, J. High Energy Phys. 10 (2012) 140.

[7] M. Holthausen, K. S. Lim, and M. Lindner, J. High Energy Phys. 02 (2012) 037; J. Elias-Miró, J. R. Espinosa, G. F. Giudice, G. Isidori, A. Riotto, and A. Strumia, Phys. Lett. B 709, 222 (2012); S. Alekhin, A. Djouadi, and S. Moch, Phys. Lett. B 716, 214 (2012).

[8] A. V. Bednyakov, B. A. Kniehl, A. F. Pikelner, and O. L. Veretin, Phys. Rev. Lett. 115, 201802 (2015).

[9] L. Di Luzio, G. Isidori, and G. Ridolfi, Phys. Lett. B 753, 150 (2016).

[10] J. R. Espinosa, G. F. Giudice, and A. Riotto, J. Cosmol. Astropart. Phys. 05 (2008) 002; O. Lebedev and A. Westphal, Phys. Lett. B 719, 415 (2013); A. Kobakhidze and A. Spencer-Smith, Phys. Lett. B 722, 130 (2013); M. Fairbairn and R. Hogan, Phys. Rev. Lett. 112, 201801 (2014); K. Enqvist, T. Meriniemi, and S. Nurmi, J. Cosmol. Astropart. Phys. 07 (2014) 025; A. Hook, J. Kearney, B. Shakya, and K. M. Zurek, J. High Energy Phys. 01 (2015) 061; M. Herranen, T. Markkanen, S. Nurmi, and A. Rajantie, Phys. Rev. Lett. 113, 211102 (2014); F. Bezrukov, J. Rubio, and M. Shaposhnikov, Phys. Rev. D 92, 083512 (2015); A. Shkerin and S. Sibiryakov, Phys. Lett. B 746, 257 (2015); J. Kearney, H. Yoo, and K. M. Zurek, Phys. Rev. D 91, 123537 (2015); P. Burda, R. Gregory, and I. Moss, J. High Energy Phys. 08 (2015) 114; C. Gross, O. Lebedev, and M. Zatta, Phys. Lett. B 753, 178 (2016); M. Herranen, T. Markkanen, S. Nurmi, and A. Rajantie, Phys. Rev. Lett. 115, 241301 (2015); L. Delle Rose, C. Marzo, and A. Urbano, arXiv:1507.06912; P. Burda, R. Gregory, and I. Moss, arXiv:1601.02152; Y. Ema, K. Mukaida, and K. Nakayama, arXiv:1602.00483; K. Kohri and H. Matsui, arXiv:1602.02100.

[11] C. D. Froggatt and H. B. Nielsen, Phys. Lett. B 368, 96 (1996); M. Shaposhnikov and C. Wetterich, Phys. Lett. B 683, 196 (2010); I. Bars, P. J. Steinhardt, and N. Turok, Phys. Lett. B 726, 50 (2013); A. Hebecker, A. K. Knochel, and T. Weigand, J. High Energy Phys. 06 (2012) 093; L. E. Ibañez, F. Marchesano, D. Regalado, and I. Valenzuela, J. High Energy Phys. 07 (2012) 195; L. E. Ibañez and I. Valenzuela, J. High Energy Phys. 05 (2013) 064; A. Hebecker, A. K. Knochel, and T. Weigand, Nucl. Phys. B874, 1 (2013); M. Ibe, S. Matsumoto, and T. T. Yanagida, Phys. Lett. B 732, 214 (2014); F. D'Eramo, L. J. Hall, and D. Pappadopulo, J. High Energy Phys. 06 (2015) 117; J. R. Espinosa, J. F. Fortin, and M. Trepanier, arXiv:1508.05343.

[12] J. R. Espinosa, G. F. Giudice, E. Morgante, A. Riotto, L. Senatore, A. Strumia, and N. Tetradis, J. High Energy Phys. 09 (2015) 174.
[13] R. Jackiw, Phys. Rev. D 9, 1686 (1974).

[14] D. Johnston, Nucl. Phys. B253, 687 (1985); G. Thompson and H. L. Yu, Phys. Rev. D 31, 2141 (1985); G. Kunstatter and H. P. Leivo, Phys. Lett. B 183, 75 (1987); J. R. S. Do Nascimento and D. Bazeia, Phys. Rev. D 35, 2490 (1987); D. Boyanovsky, D. Brahm, R. Holman, and D. S. Lee, Phys. Rev. D 54, 1763 (1996); C. Contreras and L. Vergara, Phys. Rev. D 55, 5241 (1997); 56, 6714(E) (1997); D. Boyanovsky, W. Loinaz, and R. S. Willey, Phys. Rev. D 57, 100 (1998); R. Haussling and S. Kappel, Eur. Phys. J. C 4, 543 (1998); O. M. Del Cima, D. H. T. Franco, and O. Piguet, Nucl. Phys. B551, 813 (1999); J. Baacke and K. Heitmann, Phys. Rev. D 60, 105037 (1999); L. P. Alexander and A. Pilaftsis, J. Phys. G 36, 045006 (2009).

[15] D. Metaxas and E. J. Weinberg, Phys. Rev. D 53, 836 (1996); D. Metaxas, Phys. Rev. D 63, 085009 (2001).

[16] G. Isidori, G. Ridolf,i, and A. Strumia, Nucl. Phys. B609, 387 (2001).

[17] H. H. Patel and M. J. Ramsey-Musolf, J. High Energy Phys. 07 (2011) 029.

[18] M. Garny and T. Konstandin, J. High Energy Phys. 07 (2012) 189.

[19] A. Andreassen, W. Frost, and M. D. Schwartz, Phys. Rev. D 91, 016009 (2015); Phys. Rev. Lett. 113, 241801 (2014).

[20] A. D. Plascencia and C. Tamarit, arXiv:1510.07613; Z. Lalak, M. Lewicki, and P. Olszewski, arXiv:1605.06713.

[21] S. R. Coleman and E. J. Weinberg, Phys. Rev. D7, 1888(1973).

[22] S. P. Martin, Phys. Rev. D 90, 016013 (2014).

[23] J. Elias-Miró, J. R. Espinosa, and T. Konstandin, J. High Energy Phys. 08 (2014) 034.

[24] W. Loinaz and R. S. Willey, Phys. Rev. D 56, 7416 (1997).

[25] N. K. Nielsen, Nucl. Phys. B101, 173 (1975).

[26] I. J. R. Aitchison and C. M. Fraser, Ann. Phys. (N.Y.) 156, 1 (1984).

[27] C. Ford, D. R. T. Jones, P. W. Stephenson, and M. B. Einhorn, Nucl. Phys. B395, 17 (1993); M. B. Einhorn and D. R. T. Jones, J. High Energy Phys. 04 (2007) 051.

[28] S. P. Martin, Phys. Rev. D 89, 013003 (2014).

[29] A. Pilaftsis and D. Teresi, Nucl. Phys. B906, 381 (2016); G. Markó, U. Reinosa, and Z. Szép, arXiv:1604.04193.

[30] N. Kumar and S. P. Martin, arXiv:1605.02059.

[31] C. Kim, Phys. Rev. D 90, 067701 (2014); 90, 089903(E) (2014).

[32] L. Dolan and R. Jackiw, Phys. Rev. D 9, 3320 (1974).

[33] L. Di Luzio and L. Mihaila, J. High Energy Phys. 06 (2014) 079.

[34] R. Fukuda and T. Kugo, Phys. Rev. D 13, 3469 (1976).

[35] M. Laine, Phys. Rev. D 51, 4525 (1995); K. Kajantie, M. Laine, K. Rummukainen, and M. E. Shaposhnikov, Nucl. Phys. B466, 189 (1996).

[36] D. Johnston, Phys. Lett. B 186, 185 (1987).

[37] M. Dine, P. Draper, H. E. Haber, and L. S. Haskins, arXiv:1607.06995.

[38] P. Gambino and P. A. Grassi, Phys. Rev. D 62, 076002 (2000).

[39] J. A. Casas, J. R. Espinosa, M. Quirós, and A. Riotto, Nucl. Phys. B436, 3 (1995); B439, 466(E) (1995).

[40] A. Sirlin and R. Zucchini, Nucl. Phys. B266, 389 (1986).

[41] S. R. Coleman, J. Wess, and B. Zumino, Phys. Rev. 177, 2239 (1969); C. Arzt, Phys. Lett. B 342, 189 (1995).

[42] N. K. Nielsen, Phys. Rev. D 90, 036008 (2014). 